\title{
Phase transitions in optimal betting strategies
}

\author{
L. Dinis ${ }^{1}$, J. Unterberger ${ }^{2}$ and D. Lacoste ${ }^{3}$ \\ ${ }^{1}$ GISC - Grupo Interdisciplinar de Sistemas Complejos and Dpto. de Estructura de la Materia, \\ FÃysica TÃlrmica y ElectrÃşnica, Universidad Complutense de Madrid, 28040 Spain \\ 2 Institut Elie Cartan, UMR CNRS 7502, Université de Lorraine, \\ BP 239 F-54506 Vandoeuvre-lès-Nancy Cedex, France \\ 3 Gulliver Laboratory, UMR CNRS 7083, PSL Research University, \\ ESPCI, 10 rue Vauquelin, F-75231 Paris Cedex 05, France
}

(Dated: September 1, 2020)

\begin{abstract}
Kelly's criterion is a betting strategy that maximizes the long term growth rate, but which is known to be risky. Here, we find optimal betting strategies that gives the highest capital growth rate while keeping a certain low value of risky fluctuations. We then analyze the trade-off between the average and the fluctuations of the growth rate, in models of horse races, first for two horses then for an arbitrary number of horses, and for uncorrelated or correlated races. We find an analog of a phase transition with a coexistence between two optimal strategies, where one has risk and the other one does not. The above trade-off is also embodied in a general bound on the average growth rate, similar to thermodynamic uncertainty relations. We also prove mathematically the absence of other phase transitions between Kelly's point and the risk free strategy.
\end{abstract}

PACS numbers: $05.70 . \operatorname{Ln}$, 05.40.-a 02.50.Le

\section{INTRODUCTION}

Developed in 1956 by Bell Labs scientist John Kelly, Kelly's criterion applied the newly created field of information theory to gambling and investment [1]. Largely popularized in books [2, this criterion allows a gambler (or investment fund) to fix what proportion of bankroll should be risked on a given bet. It essentially exploits side information to maximize the expected geometric growth rate of a capital. This work was precursor to the growth optimal portfolio theory, which applied these ideas to capital market [3]. The ensemble of optimal investment strategies forms an efficient border [4, or equivalently a Pareto front [5, 6], which is a term used in engineering and economics to call the set of designs that represent best trade-offs between different conflicting requirements.

Recently, there has been a surge of interest in applying insights from optimal gambling theory and economy to biology. Kelly's work led to an essential clarification of the concept of information value in biology [7, 8, which was very helpful to understand strategies used by biological systems in a fluctuating environment. In particular the bet-hedging strategy turned out to be precisely an optimal strategy of the Kelly type [9, 10.

Here, we focus on betting strategies of Kelly's type and draw inspiration from the field of Stochastic Thermodynamics, a recent branch of Thermodynamics with deep links to information theory, and with already several works specifically applied to gambling or betting prob- lems 11 14. A recent and an active line of research concerns the thermodynamic uncertainty relations [15]18, which capture important tradeoffs in Thermodynamics. In this letter, we explore novel implications of these ideas for gambling models. We emphasize at this point that a background on Stochastic Thermodynamics is not required to understand this letter, since we only rely on basic notions of probability and optimization theory.

To gain insight into the tradeoff present in gambling, we study the efficient border of Kelly's model, and we find that it extends to a region of negative growth, never discussed in the literature to our knowledge, corresponding to catastrophic betting strategies. Inspired by works on optimal protocols [19 21], and specifically on phase transitions among optimal protocols [22], we identify similar phase transitions in optimal betting strategies. We first prove such a result for uncorrelated races, and involving only two horses, which we then generalize to an arbitrary number of horses and to correlated races. In addition, we also give a general proof of the convexity in the most useful part of the front (positive part of the tradeoff branch), which rules out the existence of further phase transitions on that branch.

\section{KELLY'S HORSE RACES}

Let us recall here the main features of Kelly's horse race [1]. This race involves $M$ horses, which are numbered as $1,2 \ldots M$. The 
odds paid by the bookmaker when the horse $x$ wins is $o_{x}$, and the probability for this to happen is $p_{x}$. A gambler can distribute his bets on the different horses, let $b_{x}$ be the fraction of the bet set on horse $x$, so that $\sum_{x=1}^{M} b_{x}=1$. For all $x, b_{x}>0$, because the gambler bets on all horses but only makes money from the horse $x$ that wins.

A key feature of the model is that this dynamics is repeated, since all the money gained in one race is reinvested in the next race. Thus, the capital $C_{N+1}$ of the gambler after $N+1$ races is related to his capital after $N$ races, $C_{N}$, by the expression

$$
C_{N+1}=o_{x} b_{x} C_{N}, \text { with probability } p_{x} .
$$

The important quantity is the long term growth rate of the capital which has the form

$$
\lim _{N \rightarrow \infty} \frac{1}{N} \ln C_{N}=\sum_{x} p_{x} \ln \left(o_{x} b_{x}\right)
$$

where the equality follows from the law of large numbers. Let us introduce the random variable $W_{x}=\ln \left(o_{x} b_{x}\right)$ which describes the contribution of horse $x$ to this growth rate. Its average with respect to the probability density $p_{x}$, is the long term growth rate denoted $\langle W\rangle$.

Kelly's strategy is defined from the optimization of this average growth rate over the betting strategy defined by $b_{x}$. A simple calculation given the constraint $\sum_{x} b_{x}=1$ leads to the proportional betting strategy $b_{x}^{*}=p_{x}$. This particular solution is independent of the odds $o_{x}$, but if there was a track take, the optimal solution would depend on both $o_{x}$ and $p_{x}[1$.

Games of this type can be easily simulated in a computer using a random number generator to choose a winning horse for each race according to probability distribution $p_{x}$ and using Eq. (1) to compute gambler's capital [23]. The growth of the capital is exponential and Kelly's strategy dominates on long times all non-optimal strategies as shown in Fig. 1 .

A central result of Stochastic Thermodynamics, namely fluctuation relations, can be obtained in a few steps for this model [14. Using the definition of $W$, and given that $b=p$ for Kelly's strategy, we obtain :

$$
\left\langle e^{-W}\right\rangle=\sum_{x} p_{x} \frac{1}{o_{x} p_{x}}=\sum_{x} \frac{1}{o_{x}}=1,
$$

where in the last equality, we have used the normalization of the distribution $r_{x}=1 / o_{x}$ valid when there is no track take (fair odds). By Jensen's inequality, Eq. (3) implies $\langle W\rangle \geq 0$,

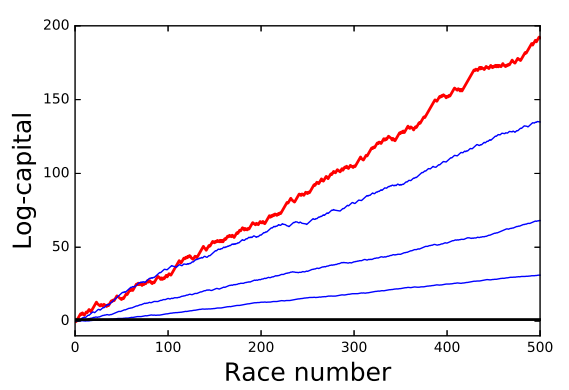

Figure 1: Logarithm of the capital of the gambler versus the number of races, for the optimal strategy (Kelly's) (thick red line) and for a selection of three non-optimal strategies (thin blue lines).

which also follows from $\langle W\rangle=D(p \mid r) \geq 0$ where $D(p \mid r)$ denotes the Kullback-Leibler divergence between the distributions $p$ and $r$. This fluctuation relation (3) can be generalized for an arbitrary strategy of the gambler, not necessarily that of Kelly, and when the odds are not necessarily fair, by introducing the decomposition $\tilde{W}_{x}=W_{x}+I_{x}$, where $W_{x}=\ln \left(o_{x} b_{x}\right)$ as above, $\tilde{W}_{x}=\ln \left(o_{x} p_{x}\right)$ and $I_{x}=\ln \left(p_{x} / b_{x}\right)$. In this way, $\tilde{W}$ represents the growth rate of the gambler according to Kelly's strategy and $I$ measures the difference between the gambler's strategy and that of Kelly's in a KL sense, since $\langle I\rangle=D(p \mid b)$. We have then

$$
\left\langle e^{-\tilde{W}}\right\rangle=\left\langle e^{-W-I}\right\rangle=\Lambda,
$$

with $\Lambda=\sum_{x} 1 / o_{x}$. In the same way that Eq. (3) is the analog of Jarzynski equality, Eq. (4) is similar to its generalization for absolutely irreversible processes [24. By Jensen's inequality, the second-law like inequality $\langle W\rangle \geq$ $-\langle I\rangle-\ln \Lambda$, follows which reduces to $\langle W\rangle \geq 0$ in the particular case of Kelly's strategy with fair odds. Note that in the general case, $\langle W\rangle$ can a priori be of any sign.

\section{MEAN-VARIANCE TRADEOFF : CHOICE OF UTILITY FUNCTION}

Kelly's strategy focuses on the maximization of the growth rate at the price of overlooking risk. Although bankruptcy is absent in Kelly's scenario because the growth of the capital is geometric instead of arithmetic, the fluctuations of the capital are large as shown in Fig. 1 and represents a major concern. This problem has been widely recognized in the gambling community. In practice gamblers and investors know that optimal Kelly can be âĂIJ- 
too riskyâĂİ; and that âĂIJfractional KellyâĂİ should be preferred, which deviates from the optimal solution but reduces the effective variance of the stochastic growth 3 .

In the same spirit, we study here the optimal betting strategy that gives the highest capital growth rate while keeping a certain low value of risky fluctuations and analyze the corresponding trade-off between risk and gain. A similar idea is behind the mean-variance analysis introduced by Markowitz optimization 4. In contrast with Markowitz optimization however, which considers the mean and variance of the capital return in one race, we consider here the mean and the variance of the (long-term) growth rate of the capital after many races. This important conceptual difference allows us to recover Kelly's point as a special case of our analysis, whereas Kelly's point could not appear as a limiting case of Markowitz's optimization for this reason. Hence, our utility function is a linear combination of the mean and standard deviation of the growth rate, namely $\langle W\rangle$ and $\sigma_{W}$ :

$$
\tilde{J}=\alpha\langle W\rangle-(1-\alpha) \sigma_{W},
$$

with $0 \leq \alpha \leq 1$. In practice, we use the modified utility function

$$
J=\alpha\langle W\rangle-(1-\alpha) \sigma_{W}+\lambda \sum_{x} b_{x},
$$

where $\lambda$ is a Lagrange multiplier associated to the normalization of the bets. An optimization of $J$ with respect to $b_{x}$ leads to $\lambda=-\alpha$. By reporting this into Eq. (6), the optimal bets $b_{x}$ are solutions of :

$$
p_{x}-b_{x}=\frac{\gamma}{\sigma_{W}} p_{x}\left[\ln \left(o_{x} b_{x}\right)-\langle W\rangle\right],
$$

where $\gamma=(1-\alpha) / \alpha$. As expected, when $\alpha=1$ $(\gamma=0)$, we recover the proportional betting of Kelly's strategy, which maximizes $\langle W\rangle$. Instead when $\alpha=0(\gamma \rightarrow \infty)$, we obtain the null strategy also called the risk free strategy, because in this case $\langle W\rangle=\sigma_{W}=0$. Between these two values, the strategy of the gambler is described as mixed since it combines aspects associated to the optimization of $\langle W\rangle$ and $\sigma_{W}$.

\section{EXACT SOLUTION FOR TWO HORSES}

Before embarking on the full problem with an arbitrary number of horses, it is instructive to analyze the fully solvable case of two horses.

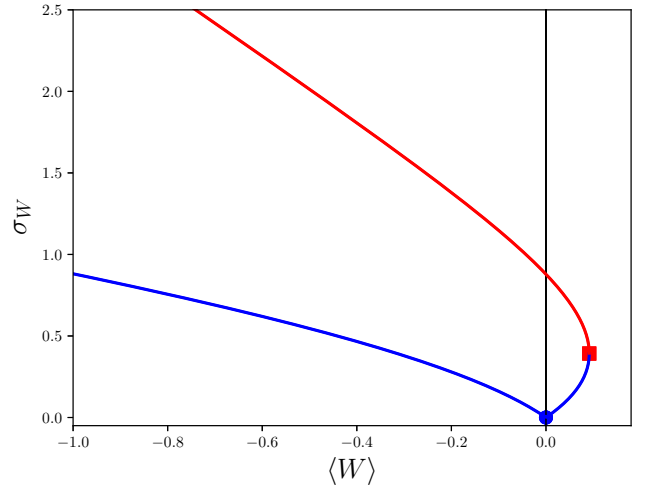

Figure 2: Trade-off branch (lower blue solid line) and non-trade-off branch (upper red solid line) in the plane $\left(\langle W\rangle, \sigma_{W}\right)$ for two horses and for the parameters $(p=0.2, r=0.4)$. The two branches meet at the red square (Kelly's strategy), and the blue circle represents the null strategy.

Let the probability that the first horse wins (resp. loses) be $p$ (resp. $1-p$ ); the bet and the odd on the first (resp. second) horse are $b$ and $1 / r$ (resp. $1-b$ and $1 /(1-r)$ ) and let us introduce the parameter $\sigma=\sqrt{p(1-p)}$.

From the optimization of $J$, we obtain the optimal strategy $b^{ \pm}$:

$$
b^{ \pm}=p \pm \gamma \sigma,
$$

where the $+($ resp. -$)$ sign corresponds to an overbetting (resp. underbetting) strategy with respect to Kelly's strategy where $b=p$.

By reporting the optimal bet given by Eq. (B3) into the expression of $J$, one obtains the efficient border. As shown in Fig. 2 this border has two branches which meet at Kelly's point. When $p<r$ the lower blue solid line is the trade-off branch associated with $b^{+}$, while the upper red solid line is the non-trade-off branch, associated with $b^{-}$. The roles of $b^{-}$and $b^{+}$exchange when instead $p>r$. Let us first focus on the region where $\langle W\rangle \geq 0$.

We find that the slope of the Pareto border is

$$
\left.\frac{d \sigma_{W}}{d\langle W\rangle}\right|_{\gamma}=\frac{\sigma}{p-b}
$$

where $b$ is equal to $b^{-}$when $r<p[23$. Therefore the slope of the Pareto border is infinite at Kelly's point where $b^{ \pm}=p$; while it reaches a finite value near the null strategy, namely

$$
\left.\frac{d \sigma_{W}}{d\langle W\rangle}\right|_{\gamma_{c}}=\frac{1}{\gamma_{c}}=\frac{\sigma}{|p-r|} .
$$


This signals a phase transition at this critical value $\gamma_{c}$, where the optimal strategy changes from the null strategy to a mixed strategy. As a result, the optimal $J$ versus $\gamma$ changes from zero when $\gamma \geq \gamma_{c}$ (null strategy) to a non-zero value when $\gamma \leq \gamma_{c}$ (mixed strategy). For two horses, such a plot is similar to what is shown for three horses in the inset of Fig. 3

To prove the existence of the phase transition, we have checked that the border is convex near the null strategy. It is indeed the case since

$$
\left.\frac{d^{2} \sigma_{W}}{d\langle W\rangle^{2}}\right|_{\gamma=\gamma_{c}}=\frac{r(1-r)}{\sigma^{2} \gamma_{c}^{3}}>0 .
$$

In the rest of this paper, we now focus on the general case for an arbitrary number of horses.

\section{NUMERICAL RESULTS}

Let us now explain how to obtain the Pareto front from a numerical optimization of the utility function using a simulated annealing algorithm, as illustrated in Fig. 3 for the case of three horses. Similarly to the case of two horses case, the lower and upper branch correspond to different optimization problems. The lower branch is formed by bets that maximize the growth rate $\langle W\rangle$ for a given value of the fluctuations $\sigma_{W}$, whereas the upper branch corresponds to maximal fluctuations $\sigma_{W}$ for a given value of the growth rate $\langle W\rangle$.

For the lower branch, there are two regions where $\langle W\rangle$ is either positive or negative. In the former case, the front is convex and can be recovered by the maximization of the utility function $J=J_{1}$ defined in Eq. (5). In contrast, in the negative $\langle W\rangle$ region, the front is concave and a different strategy is needed. Following 22 , we use a quadratic objective function

$$
J_{2}=-\left(\langle W\rangle-W_{0}\right)^{2}-k \sigma_{W}
$$

We use a global minus sign in order to keep the same maximization procedure, although we wish in fact to minimize both the value of $\sigma_{W}$ and the distance to a target value $W_{0}$ for the growth rate. By varying the target value $W_{0}$ from 0 to a sufficiently negative value we can draw the negative lower branch. Parameter $k$ weighs the importance between the constraint of $\langle W\rangle$ being close to $W_{0}$ or minimizing the value of the fluctuations. We took $k=0.5$ although other moderate values would do.

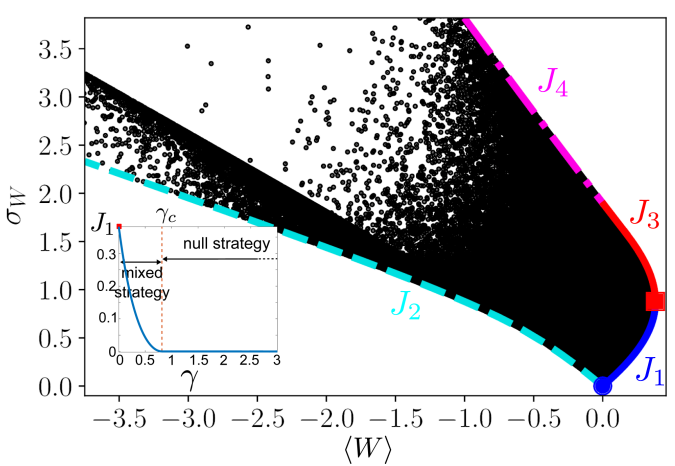

Figure 3: Pareto borders for 3 horses obtained from numerical optimization of the utility functions $J_{1}, J_{2}, J_{3}$ and $J_{4}$ (colored solid lines), together with a cloud of points generated by randomly choosing bets satisfying all relevant constraints. Parameters are $p_{1}=0.2, p_{2}=0.6$, $r_{1}=0.4$ and $r_{2}=0.2$ for the first two horses. Inset: $J_{1}$ versus $\gamma$ along the trade-off branch (i.e. on the dark blue border).

Similarly, the upper branch with positive $\langle W\rangle$ is concave and corresponds to the maximization of the objective function

$$
J_{3}=\alpha\langle W\rangle+(1-\alpha) \sigma_{W},
$$

where the plus sign before $\sigma_{W}$ now ensures the maximization of the fluctuations in contrast with the lower branch case. The upper branch with negative $\langle W\rangle$ appears almost straight for large negative values of $\langle W\rangle$. Thus, although $J_{3}$ could still be used there, further numerical precision can be achieved by using a modified objective function

$$
J_{4}=-\left(\langle W\rangle-W_{0}\right)^{2}+k \sigma_{W}
$$

where again the plus sign in front of $\sigma_{W}$ corresponds to the maximization of fluctuations.

General conclusions can also be obtained for this model near special points. Near Kelly's point, we find that the slope of the Pareto border is always vertical. This means that in practice if one is willing to pay a sacrifice a small amount of the average growth rate, one can lower the fluctuations significantly, thereby accessing "safer" strategies such as the blue curves in Fig. 1 Near the null strategy, we find a similar phase transition as in the two horses case, which we now analyze in more details. 


\section{MEAN-VARIANCE TRADE-OFF : BOUNDS}

We recall that $r_{x}:=1 / o_{x}$ and we assume a fair game for which $\sum_{x} r_{x}=1$. Then let $q_{x}:=r_{x} / p_{x}$, so that the first two moments of $q$ are $\langle q\rangle=1$ and $\sigma_{q}^{2}:=\left\langle q^{2}\right\rangle-\langle q\rangle^{2}=\left\langle q^{2}\right\rangle-1$. Let us focus on the branch of positive $\langle W\rangle$. In this case, we find the following inequality,

$$
\sigma_{W} \geq \frac{\langle W\rangle}{\sigma_{q}}
$$

which has a similar structure as thermodynamic uncertainty relations [15, 25], and which captures a general trade-off between the mean and the variance of the growth rate.

The proof goes as follows: we consider the quantity $\sigma_{q}^{2} \sigma_{W}^{2}$, since $\sigma_{q}^{2}=\left\langle q^{2}\right\rangle-1$, we have using the Cauchy-Schwarz inequality

$$
\begin{aligned}
\sigma_{q}^{2} \sigma_{W}^{2} & =\left\langle(q-1)^{2}\right\rangle\left\langle(W-\langle W\rangle)^{2}\right\rangle, \\
& \geq\langle(q-1)(W-\langle W\rangle)\rangle^{2} \\
& \geq(\langle q W\rangle-\langle W\rangle)^{2}
\end{aligned}
$$

Now since $\langle q W\rangle=\sum_{x} r_{x} / b_{x} \log \left(b_{x} / r_{x}\right)=$ $-D(r \mid b) \leq 0$, then Eq.(15) follows. This inequality is saturated when $b_{x}=r_{x}$, which corresponds to the null strategy.

Similar inequalities can be derived using instead other relevant Kullback-Leibler divergences, such as $D(b \mid p)$ or $D(r \mid p)$. To exploit the first divergence, we introduce the ratio $s_{x}=b_{x} / p_{x}$ which is also a normalized probability distribution similar to $q$, with a second moment $\sigma_{s}^{2}$. Then, following the same steps, we obtain an inequality for the quantity $I$ introduced in $\mathrm{Eq}(3)$ :

$$
\sigma_{I} \geq \frac{\langle I\rangle}{\sigma_{s}}
$$

which is saturated when $b_{x}=p_{x}$, i.e. for Kelly's strategy. To exploit the second divergence, we now use the quantity $\tilde{W}$, and we obtain the inequality

$$
\sigma_{\tilde{W}} \geq \frac{\langle\tilde{W}\rangle}{\sigma_{q}}
$$

which is saturated when $p_{x}=r_{x}$. Note that Eqs. 17 and 18 represent new bounds which complement the inequalities $\langle I\rangle \geq 0$ and $\langle\tilde{W}\rangle \geq$ 0 obtained previously.

\section{PHASE TRANSITION IN OPTIMAL STRATEGIES}

In order to prove that there are no tighter bounds of this type, we carry out a perturbation calculation near the null strategy using the vector $\epsilon_{x}$

$$
o_{x} b_{x}=\frac{b_{x}}{r_{x}}=1+\varepsilon_{x} .
$$

To ensure that $\vec{b}$ is still a probability measure, we require that the column vector $\vec{\varepsilon}=\left(\varepsilon_{x}\right)_{x}$ lies on the hyperplane $(\vec{r}, \vec{\varepsilon})=\sum_{x} r_{x} \varepsilon_{x}=0$.

By evaluating $\langle W\rangle$ and $\sigma_{W}$ to first order in $\vec{\varepsilon}$, we find that $\sigma_{W} \sim\langle W\rangle / \gamma_{c}$, with

$$
\gamma_{c}=\sigma_{q}
$$

an expression which we can be checked by plotting a zoom of the Pareto border near the null strategy 23. The evaluation of the second order derivative at the null strategy on the Pareto border requires a calculation to second order in $\vec{\varepsilon}$, which gives

$$
\left.\frac{d^{2} \sigma_{W}}{d\langle W\rangle^{2}}\right|_{\gamma=\gamma_{c}}=\frac{C}{\gamma_{c}^{5}},
$$

where $C=\left\langle q^{3}\right\rangle-\left\langle q^{2}\right\rangle^{2}[23$. By Cauchy-Schwarz again, it follows that $\left\langle q^{2}\right\rangle^{2}=\left\langle q^{3 / 2} q^{1 / 2}\right\rangle^{2} \leq\left\langle q^{3}\right\rangle$, thus $C \geq 0$, with equality iff $p_{x}=r_{x}$.

In the particular case of two horses, it is straightforward to check that the expression of $\gamma_{c}$ given in Eq. (10) and that of the second derivative in Eq. (B13) are recovered from Eqs. (B9)-(D14). These calculations show that there is always a phase transition in this model near the null strategy for an arbitrary number of horses in the region of positive $\langle W\rangle$. A similar calculation shows that the slope has the opposite value on the other side in the region of negative $\langle W\rangle$.

\section{SHAPE OF THE FRONT : GENERAL RESULTS}

\section{A. Large negative growth rate}

In the regions of the phase diagram corresponding to negative values of $\langle W\rangle$, the Pareto front is open. Namely, the growth rate diverges because it is evaluated on some $b_{x} \rightarrow 0$. Easy computations shows that points in the $\left(\langle W\rangle, \sigma_{W}\right)$ plane satisfy asymptotically $\langle W\rangle \rightarrow$ $-\infty$ and $\sigma_{W} /\langle W\rangle \rightarrow-\sqrt{\left(1-P^{\prime}\right) / P^{\prime}}$ when 
bets $b_{x^{\prime}} \rightarrow 0$ for $x^{\prime} \in X^{\prime}$ with $P^{\prime}:=\sum_{x^{\prime} \in X^{\prime}} p_{x^{\prime}}$. The smallest slope (lower front), is obtained by putting all the bets on the horse $x^{*}$ which has the least chances to win; this is the worst strategy.

\section{B. Lower front : positive growth rate}

In order to decide whether other phase transitions are possible in this model, we now study the convexity of the front near any point. More precisely, we define the front as the extremum locus of the functional

$$
\begin{gathered}
\tilde{J}_{m^{*}}(b ; \lambda, \mu):=\left\langle W^{2}\right\rangle+\lambda\left(\langle W\rangle-m^{*}\right) \\
+2 \mu\left(\sum_{x} b_{x}-1\right)
\end{gathered}
$$

where $\lambda, \mu$ are Lagrange multipliers fixing $\langle W\rangle$ and implementing the bet normalization constraint. The procedure is equivalent to extremizing the variance for a given average value $m^{*}$. The null gradient condition $D \tilde{J}_{m^{*}}(b ; \lambda, \mu)=0$ defines $(b, \lambda, \mu)$ as an implicit function $f\left(m^{*}\right)$ of $m^{*}$. The gradient of $f$, which is the Hessian of $\tilde{J}_{m^{*}}$, may be inverted with some efforts, yielding by the implicit function theorem the slope $d \sigma_{W} / d\langle W\rangle=d \sigma_{W} / d m^{*}$ and then finally, the second derivative $d^{2} \sigma_{W} / d\langle W\rangle^{2}$ in terms of $\mu$ (proportional to the inverse of the Pareto slope parameter $\gamma$ ) and averaged functionals of $b_{x} / p_{x}$. Explicit formulas given in Supp. Mat 23 have been checked numerically. One can then prove in whole generality that the part of the lower front between the null strategy and Kelly's strategy is convex, turning to concave in some neighborhood of the null strategy when $\langle W\rangle<0$, and some neighborhood of Kelly's strategy on the upper front, as confirmed numerically in Fig. 2 and Fig. 3 in the case of two and three horses. Note that this calculation does not exclude the possibility of other phase transitions in other parts of the front.

\section{CORRELATED RACES}

As a variation on Kelly's horse races, we now assume that the races are no longer independent but follow from an ergodic Markov process defined by the conditional probability $p_{x \mid y}$, which represents the probability that the horse $x$ wins if the previous horse that won the race was horse $y$. Let the bets be also conditional and defined by $b_{x \mid y}$ such that $\sum_{x} b_{x \mid y}=1$. The odds denoted by $o_{x}=1 / r_{x}$ are assumed to be fair $\sum_{x} r_{x}=1$. The average growth rate $\langle W\rangle$ now takes the following form

$$
\langle W\rangle=\lim _{N \rightarrow \infty}\left\langle W_{N}\right\rangle=\sum_{x, y} p_{x \mid y} \bar{p}_{y} \ln \left(b_{x \mid y} o_{x}\right),
$$

where $\bar{p}_{y}$ denote the unique steady state probability of the races. By optimizing $\langle W\rangle$ with respect to $b_{x \mid y}$, we find that the optimal strategy is still proportional betting with now $p_{x \mid y}=b_{x \mid y}$. This is the new Kelly's strategy for this case.

On the trade-off branch, the relevant utility function is

$$
J=\alpha\langle W\rangle-(1-\alpha) \sigma_{W}+\sum_{y} \lambda_{y} \sum_{x} b_{x \mid y},
$$

where $\lambda_{y}$ are Lagrange multipliers associated to the normalization of the bets. The Pareto borders are shown in Fig. 4. We observe numerically that when correlations are present the upper front for negative $\mathrm{W}$ becomes convex in some intermediate region. In that region, the border can not longer be described by $J_{3}$ and the use of $J_{4}$ is unavoidable.

The null strategy corresponds to the condition that for any $x, y, b_{x \mid y}=r_{x}$, in which case both the average growth rate and its variance are zero. An expansion with respect to that strategy can be carried as before. The $q$ distribution is now defined as $q_{x \mid y}=r_{x} / p_{x \mid y}$, which is a probability distribution because

$$
\langle q\rangle=\sum_{x y} p_{x \mid y} \bar{p}_{y} \frac{r_{x}}{p_{x \mid y}}=\sum_{x y} \bar{p}_{y} r_{x}=1 .
$$

Its second moment is now $\left\langle q^{2}\right\rangle=\sum_{x y} p_{x \mid y} \bar{p}_{y} q_{x \mid y}^{2}$. Except for this modification, the critical $\gamma$ takes the same form as in Eq. $\overline{\mathrm{B} 9}$, which is numerically tested in the inset of Fig. 4 .

An inequality similar to Eq. (15) can also be obtained in the case of correlated races because in this case the conditional bets $b_{x \mid y}$ are still a probability distribution $\sum_{x} b_{x \mid y}=1$, and therefore following the same steps, the positivity of $D(r \mid b)$ leads to a similar result. In fact, the normalization of $q$ is equivalent to a fluctuation relation generalizing Eq. (4)[14]. because in that case

$$
\left\langle e^{-W}\right\rangle=\sum_{x y} p_{x \mid y} \bar{p}_{y} \frac{1}{p_{x \mid y} o_{x}}=1,
$$

while

$$
\left\langle e^{-W-I}\right\rangle=\Lambda
$$

holds in the general case for an arbitrary strategy with $I_{x \mid y}=\ln \left(p_{x \mid y} / b_{x \mid y}\right)$. 


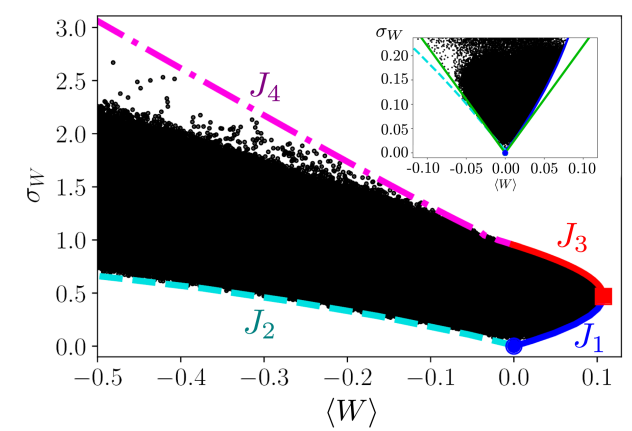

Figure 4: Same plot as in fig. 3 but for the case of for 3 horses in the presence of correlations between the races. Parameters are detailed in Ref. 23]. Inset: zoom near the null strategy together with predictions from linear approximation.

\section{CONCLUSION}

In this work, we have derived general Fluctuation Relations for betting models of Kelly's type, and a bound on the average capital growth rate, similar to thermodynamic uncertainty relations. This bound captures the classic trade-off between average growth rate and risk, which plays a central role in money investment [26]. In models with repetitive investment dynamics, all utility functions become under suitable conditions equivalent to a utility function with a log mean variance form [3], which is the form considered here. This suggests that our work should be applicable to a broad class of econophysics models, for which log utility functions are used.

In our work, we have identified a phase transition between the null strategy and a mixed strategy, and we have shown that there is no other phase transition between the null strategy and Kelly's point due to the convexity of the lower front. We have also illustrated how to handle non-convex utility functions, an important issue for applications to machine learning 27 .

The explicit analytical expressions which we have obtained for the slope and curvature of the front at any point could be used to move directly along the front, as an alternative to the involved optimization algorithm used here. It would be also interesting to explore more systematically how additional constraints affect the efficient border. The question of adaptative optimization of the bets, where possible nonMarkovian or non-ergodic features could arise, is a rich inference problem worth pursuing [10]. Finally, we hope that this framework could open news research directions on evolutionary tradeoffs and Pareto optimality in biology [5, 6].

\section{ACKNOWLEDGMENTS}

L.D. acknowledges financial support from Spanish Ministerio de EconomÃna, Industria y Competitividad through grant FIS2017-83709R. We acknowledge many insightful discussions with L. Peliti and E. Aurell.
[1] J. L. J. Kelly, Bell System Technical Journal 35, 917 (1956).

[2] W. Poundstone, Fortune's formula (Hill and Wang, 2005).

[3] L. C. MacLean, E. O. Thorp, and W. T. Ziemba, Kelly capital growth investment criterion, the theory and practice (Word Scientific, 2011).

[4] H. Markowitz, J. of Finance 7, 77âĂŞ91 (1952).

[5] L. F. Seoane and R. Solé, Phys. Rev. E 92, 032807 (2015).

[6] O. Shoval, H. Sheftel, G. Shinar, Y. Hart, O. Ramote, A. Mayo, E. Dekel, K. Kavanagh, and U. Alon, Science 336, 1157 (2012).

[7] C. T. Bergstrom and M. Lachmann, in Information Theory Workshop (2004) pp. 50-54.

[8] O. Rivoire and S. Leibler, J. Stat. Phys. 142, 1124 (2011).

[9] E. Kussell and S. Leibler, Science 309, 2075 (2005).
[10] O. Tal and T. D. Tran, Bull Math Biol 82, 50 (2020).

[11] I. Neri, É. Roldán, S. Pigolotti, and F. JÃijlicher, Journal of Statistical Mechanics: Theory and Experiment 2019, 104006 (2019).

[12] S. Ito, Scientific reports 6, 36831 (2016).

[13] D. A. Vinkler, H. H. Permuter, and N. Merhav, J. Stat. Mech: Theory Exp. 2016, 043403 (2016).

[14] Y. Hirono and Y. Hidaka, J. Stat. Phys. 161, $721(2015)$.

[15] J. M. Horowitz and T. R. Gingrich, Nat. Physics , 1 (2019).

[16] G. Falasco, M. Esposito, and J.-C. Delvenne, New J. of Phys. (2020).

[17] H. Vroylandt, D. Lacoste, and G. Verley, J. Stat. Mech. , 023205 (2018).

[18] D. L. K. Proesmans and L. Peliti, A case study of thermodynamic bounds for chemical kinetics (World Scientific Publishers, 2018) Chap. 
Chemical kinetics beyond the textbook.

[19] E. Aurell, C. Mejía-Monasterio, and P. Muratore-Ginanneschi, Phys. Rev. Lett. 106, 250601 (2011)

[20] H. Then and A. Engel, Phys. Rev. E 77, 041105 (2008).

[21] T. Schmiedl and U. Seifert, Phys. Rev. Lett. 98, 108301 (2007).

[22] A. P. Solon and J. M. Horowitz, Phys. Rev. Lett. 120, 180605 (2018)

[23] See Supplemental Material for details on simulations, on the exact solution for two horses, and on the analysis of the Pareto front.

[24] Y. Murashita, K. Funo, and M. Ueda, Phys. Rev. E 90, 042110 (2014).

[25] J. Uffink and J. Van Lith, Found. of Physics 29, 655 (1999).

[26] J. P. Bouchaud and M. Potters, Theory of financial risk and derivative pricing (Cambridge University Press, 2009).

[27] L. Bottou, F. E. Curtis, and J. Nocedal, Optimization methods for large-scale machine learning (2016), arXiv:1606.04838 [stat.ML].

\section{Supplementary Material}

\section{Appendix A: Notations.}

Let $r_{x}:=1 / o_{x}, W_{x}:=\ln \left(o_{x} b_{x}\right)=\ln \left(b_{x} / r_{x}\right), q_{x}:=\frac{r_{x}}{p_{x}}$. Denote by $\langle\cdot\rangle$ the average of a function $f=\left(f_{x}\right)_{x}$ with respect to the weights $\left(p_{x}\right)_{x},\langle f\rangle:=\sum_{x} p_{x} f_{x}$. Then the average growth rate is

$$
\langle W\rangle=\sum_{x} p_{x} \ln \left(b_{x} / r_{x}\right)
$$

and the standard deviation of a given strategy $\vec{b}=\left(b_{x}\right)_{x}$ is

$$
\begin{aligned}
\sigma_{W} & :=\sqrt{\langle W\rangle^{2}-\langle W\rangle^{2}} \\
& =\sqrt{\sum_{x} p_{x} \ln ^{2}\left(b_{x} / r_{x}\right)-\langle W\rangle^{2}} .
\end{aligned}
$$

By hypothesis, the column vectors $\vec{p}=\left(p_{x}\right)_{x}, \vec{r}=\left(r_{x}\right)_{x}$ and $\vec{b}=\left(b_{x}\right)_{x}$ are probability distributions, $\sum_{x} p_{x}=\sum_{x} r_{x}=\sum_{x} b_{x}=1$. Furthermore, the two first moments of $q$ are

$$
\langle q\rangle=1, \quad \sigma_{q}^{2}:=\left\langle q^{2}\right\rangle-\langle q\rangle^{2}=\left\langle q^{2}\right\rangle-1 .
$$

\section{Appendix B: Solution for two horses}

Let the probability that the first horse wins (resp. loses) be $p$ (resp. $1-p$ ); the bet and the odd on the first (resp. second) horse are $b$ and $1 / r$ (resp. $1-b$ and $1 /(1-r))$ and let us introduce the parameter $\sigma=\sqrt{p(1-p)}$. In this way, the odds are fair. Let also $\gamma:=\frac{1-\alpha}{\alpha}$ and $\sigma:=\sqrt{p(1-p)}$. Then

$$
\langle W\rangle=p \ln \left(\frac{b}{r}\right)+(1-p) \ln \left(\frac{1-b}{1-r}\right),
$$

and

$$
\sigma_{W}^{2}=p(1-p) \ln ^{2} \frac{b(1-r)}{(1-b) r}=\left(\sigma \ln \frac{b(1-r)}{(1-b) r}\right)^{2} .
$$

From the optimization of the utility function $J$ defined in the main text, we obtain the optimal strategy $b^{ \pm}$:

$$
b^{ \pm}=p \pm \gamma \sigma,
$$


where the + (resp. - ) sign corresponds to an overbetting (resp. underbetting) strategy with respect to Kelly's strategy where $b=p$. As shown in Fig. 2 of main text, these two solutions form the two branches of the efficient border which meet at Kelly's point. When $p<r$ the lower blue solid line is the tradeoff branch associated with $b^{+}$, while the upper red solid line is the non-tradeoff branch, associated with $b^{-}$. The roles of $b^{-}$and $b^{+}$exchange when instead $p>r$. Let us first focus on the region where $\langle W\rangle \geq 0$ and let us assume e.g. $p>r$, in which case

$$
b=p-\gamma \sigma .
$$

Using Eqs. B1, B2, we find

$$
\frac{d\langle W\rangle}{d b}=\frac{p-b}{b(1-b)}=\frac{\gamma \sigma}{b(1-b)},
$$

and

$$
\frac{1}{2} \frac{d\left(\sigma_{W}^{2}\right)}{d b}=\frac{\sigma^{2}}{b(1-b)} \ln \left(\frac{b(1-r)}{(1-b) r}\right) .
$$

Hence by taking the ratio of Eq. B6 and (B5),

$$
\frac{1}{2} \frac{d\left(\sigma_{W}^{2}\right)}{d\langle W\rangle}=\frac{\sigma}{\gamma} \ln \left(\frac{b(1-r)}{(1-b) r}\right)=\frac{\sigma_{W}}{\gamma}>0 .
$$

Using the definition of $\gamma$, we deduce that the slope of the Pareto border is

$$
\left.\frac{d \sigma_{W}}{d\langle W\rangle}\right|_{\gamma}=\frac{\sigma}{p-b}
$$

where $b$ is equal to $b^{-}$since we have assumed $r<p$. This equation shows that the slope becomes infinite at Kelly's point where $\gamma \rightarrow 0$ and $b^{-} \rightarrow p$; while it reaches a finite value near the null strategy, namely

$$
\left.\frac{d \sigma_{W}}{d\langle W\rangle}\right|_{\gamma_{c}}=\frac{1}{\gamma_{c}}=\frac{\sigma}{p-r} .
$$

This suggests that there is a phase transition between the null strategy and a mixed strategy at this critical value $\gamma_{c}$. To confirm this point, we need to check that the border is convex near the null strategy.

To do so, we take the derivative of Eq. B7 with respect to $\langle W\rangle$ as before :

$$
\frac{1}{2} \frac{d^{2}\left(\sigma_{W}^{2}\right)}{d\langle W\rangle^{2}}=\frac{\frac{\sigma}{\gamma} \frac{d}{d b} \ln \left(\frac{b}{1-b}\right)+\frac{\sigma_{W}}{\sigma \gamma^{2}}}{d\langle W\rangle / d b}=\frac{1}{\gamma^{2}}+b(1-b) \frac{\sigma_{W}}{\sigma^{2} \gamma^{3}} ;
$$

Finally, using the general formula

$$
(\sqrt{f})^{\prime \prime}=\frac{1}{2 \sqrt{f}} f^{\prime \prime}-\frac{1}{4} f^{-3 / 2}\left(f^{\prime}\right)^{2},
$$

we find the simple result

$$
\frac{d^{2} \sigma_{W}}{d\langle W\rangle^{2}}=\frac{b(1-b)}{\sigma^{2} \gamma^{3}}
$$

which is always positive, in particular near the null strategy where it takes the value

$$
\left.\frac{d^{2} \sigma_{W}}{d\langle W\rangle^{2}}\right|_{\gamma=\gamma_{c}}=\frac{r(1-r)}{\sigma^{2} \gamma_{c}^{3}}>0
$$




\section{Appendix C: General expansion near the null strategy to first order}

Let us now analyze the general case for an arbitrary number of horses. As already observed for the two horses case, the lower and upper branch correspond to different optimization problems. The lower branch is formed by bets that maximize the growth rate $\langle W\rangle$ with the minimal average fluctuations $\sigma_{W}$ whereas the upper branch corresponds to maximal fluctuations $\sigma_{W}$ for a given value of the growth rate $\langle W\rangle$. For the lower branch, there are two regions where $\langle W\rangle$ is either positive or negative. In the former case, the front is convex and can be recovered by the maximization of the utility function $J=J_{1}$ defined in the main text.

General conclusions can be obtained for this model near special points. Near Kelly's point, we find that the slope of the Pareto border is always vertical. To prove this, we rely on perturbation calculations near these specific strategies. In this case, we find that the first order correction to $\langle W\rangle$ vanishes, while that of $\sigma_{W}$ does not vanish. It follows from this that the slope of the border $\left(\langle W\rangle, \sigma_{W}\right)$ is indeed vertical near Kelly's point.

We now detail the expansion near the null strategy, where we find a similar phase transition as found in the two horses case. Let us introduce the vector $\epsilon_{x}$ to measure the distance to the null strategy as

$$
o_{x} b_{x}=\frac{b_{x}}{r_{x}}=1+\varepsilon_{x}
$$

To ensure that $\vec{b}$ is still a probability measure, we require that the column vector $\vec{\varepsilon}=\left(\varepsilon_{x}\right)_{x}$ lies on the hyperplane $(\vec{r}, \vec{\varepsilon})=\sum_{x} r_{x} \varepsilon_{x}=0$.

By evaluating $\langle W\rangle$ and $\sigma_{W}$, we find that

$$
\langle W\rangle \sim\langle\varepsilon\rangle-\frac{1}{2}\left\langle\varepsilon^{2}\right\rangle,
$$

and

$$
\sigma_{W}^{2} \sim\left\langle\varepsilon^{2}\right\rangle-\langle\varepsilon\rangle^{2}
$$

To leading order in $\varepsilon$ or in $\sigma_{W}$, the equation for the optimal bets, namely Eq. (8) of the main text yields

$$
\varepsilon_{x}-\langle\varepsilon\rangle \sim \frac{\sigma_{W}}{\gamma}\left(1-q_{x}\right)
$$

Multiplying (C4) by $r_{x}$ and summing over $x$ to eliminate $\varepsilon_{x}$ yields

$$
\langle\varepsilon\rangle \sim \frac{\sigma_{W}}{\gamma}\left(\left\langle q^{2}\right\rangle-1\right)
$$

But we can also use Eq. C4 to obtain to leading order in $\varepsilon$,

$$
\left\langle\varepsilon^{2}\right\rangle-\langle\varepsilon\rangle^{2} \sim \frac{\sigma_{W}^{2}}{\gamma^{2}} \sigma_{q}^{2} .
$$

Combining these two equations, we obtain the slope on the tradeoff branch near the null strategy

$$
\sigma_{W} \sim \frac{\langle W\rangle}{\gamma_{c}}=\frac{\langle W\rangle}{\sigma_{q}} .
$$

\section{Appendix D: General expansion near the null strategy to second order}

Before embarking on the evaluation of the second order derivative near the null strategy on the Pareto border, it is useful to formalize the general problem of minimization of the variance, not necessarily near the null strategy. In general the minimization of the variance leads to the optimal 
bet $b^{*}=b(m)$ parametrized by a given value of the average, $\langle W\rangle=m$. Below, we focus on the case where $m \geq 0$. The following parametrization of the bets is then appropriate to explore the space of parameters $b$ around $b(m)$ :

$$
\frac{b_{x}}{r_{x}}=\frac{b_{x}^{*}}{r_{x}}+\varepsilon_{x},
$$

coinciding with (C1) when $b^{*}=r$ is the null strategy. To simplify the notation, we will drop the subscript with the star on the $b_{x}$ and on $m$, since it will be implicit that we consider this optimal solution.

Taking into account the constraints $\langle W\rangle=m$ and $(\vec{r}, \vec{\varepsilon})=0$, this is equivalent to minimizing the functional $-\tilde{J}_{m}$ (equivalent to maximizing $\tilde{J}_{m}$ )

$$
-\tilde{J}_{m}(\varepsilon ; \lambda, \mu):=\left\langle W^{2}\right\rangle+\lambda(\langle W\rangle-m)+2 \mu(\vec{r}, \vec{\varepsilon}) .
$$

Thus, we require the null gradient condition $\tilde{\nabla} \tilde{J}_{m}=0$, where $\tilde{\nabla}:=\left(\begin{array}{c}\nabla \\ \partial_{\lambda} \\ \partial_{\mu}\end{array}\right)$, and $\nabla=\left(\partial_{\varepsilon_{x}}\right)_{x}$.

The general formula for minimization with constraints may be found in standard textbooks, implying positivity of $\mathcal{H}=-\nabla^{2} \tilde{J}_{m}=\nabla^{2}\left\langle W^{2}\right\rangle+\lambda \nabla^{2}(\langle W\rangle-m)+2 \mu \nabla^{2}(\vec{r}, \vec{\varepsilon})$. Computations yields for the null gradient condition,

$$
-\frac{1}{2} \nabla \tilde{J}_{m}=\left\{\ln \left(b_{x} / r_{x}\right)+\frac{\lambda}{2}\right\} \frac{r_{x}}{b_{x}} p_{x}+\mu r_{x}=0 .
$$

After multiplying Eq. (D3) by $b_{x} / r_{x}$ and summing over ${ }_{x}$, one obtains

$$
-\frac{\lambda}{2}=m+\mu
$$

Thus $b=b(m)$ is given by two conditions:

$$
\left\{\begin{array}{l}
\ln \left(\frac{b_{x}}{r_{x}}\right)+\mu\left(\frac{b_{x}}{p_{x}}-1\right)=m \\
\sum_{x} b_{x}=1
\end{array}\right.
$$

The r.-h. s. of the first line of (D5), a constant, is fixed by averaging, yielding the trivial relation $\langle W\rangle=m$, whence the need for an extra condition given by the second line, which defines a function $\mu(m)$. By comparing Eq. D5 with the equation for the optimal bets given in Eq. 8 of the main text, we obtain the general expression :

$$
\mu=\frac{\sigma_{W}}{\gamma}
$$

On the Pareto front, the differential of $\tilde{J}$ defined in Eq. 6 of the main text must vanish. This leads to the condition $d\langle W\rangle-\gamma d \sigma_{W}=0$, which means that $d \sigma_{W} / d m=1 / \gamma$. Then, using Eq. D6. we obtain the equally general result

$$
\mu=\frac{1}{2} \frac{d \sigma_{W}^{2}}{d m}
$$

Let us now focus on the expansion near the null strategy. We already now from the expression for the slope of the border derived in the previous section, namely Eq. C7 that $\frac{d \sigma_{W}}{d m} \rightarrow_{m \rightarrow 0} \frac{1}{\sigma_{q}}$. Let us further assume that $\frac{d^{2} \sigma_{W}}{d m^{2}} \rightarrow_{m \rightarrow 0} \tilde{C}$ where $\tilde{C}$ or equivalently $C$ given by $C \equiv \sigma_{q}^{5} \tilde{C}$ is an unknown coefficient to be determined self-consistently which controls the curvature of the border near the null strategy.

Now using Eq. D7.

$$
\frac{d \mu}{d m}=\frac{1}{2} \frac{d^{2} \sigma_{W}^{2}}{d m^{2}}=\left(\frac{d \sigma_{W}}{d m}\right)^{2}+\sigma_{W} \frac{d^{2} \sigma_{W}}{d m^{2}} \sim\left(\frac{1}{\sigma_{q}}+m \tilde{C}\right)^{2}+\tilde{C} m / \sigma_{q} \sim \frac{1}{\sigma_{q}^{2}}+3 \frac{\tilde{C}}{\sigma_{q}} m .
$$


Since to dominant order, $m \sim \sigma_{q}^{2} \mu$, we have

$$
m \sim \sigma_{q}^{2} \mu\left(1-\frac{3}{2} \tilde{C} \sigma_{q}^{3} \mu\right)
$$

We now evaluate $\varepsilon_{x}$ to second order, using $b_{x} / r_{x}=1+\varepsilon_{x}$ in Eq. D5 This yields :

$$
\varepsilon_{x}-\frac{\varepsilon_{x}^{2}}{2}+\mu\left[\frac{r_{x}}{p_{x}}\left(1+\varepsilon_{x}\right)-1\right] \sim \sigma_{q}^{2} \mu\left(1-\frac{3}{2} \tilde{C} \sigma_{q}^{3} \mu\right) .
$$

Thus $\varepsilon_{x} \sim\left(1+\sigma_{q}^{2}-\frac{r_{x}}{p_{x}}\right) \mu+O\left(\mu^{2}\right)$ to dominant order, which gives

$$
\begin{aligned}
\varepsilon_{x} & \sim\left(1+\sigma_{q}^{2}-\frac{r_{x}}{p_{x}}\right) \mu+\frac{\varepsilon_{x}^{2}}{2}-\mu \frac{r_{x}}{p_{x}} \varepsilon_{x}-\frac{3}{2} \tilde{C} \sigma_{q}^{5} \mu^{2} \\
& \sim\left(1+\sigma_{q}^{2}-\frac{r_{x}}{p_{x}}\right) \mu+\left\{\frac{1}{2}\left(1+\sigma_{q}^{2}-\frac{r_{x}}{p_{x}}\right)\left(1+\sigma_{q}^{2}-3 \frac{r_{x}}{p_{x}}\right)-\frac{3}{2} C\right\} \mu^{2}+O\left(\mu^{3}\right) .
\end{aligned}
$$

This expansion must be consistent with the condition $\sum_{x} r_{x} \varepsilon_{x}=0$. Using the above formula, one finds that the first order term vanishes, and the second order term vanishes only if and only if

$$
\frac{1}{2}\left\langle\left(1+\sigma_{q}^{2}-\frac{r_{x}}{p_{x}}\right)\left(1+\sigma_{q}^{2}-3 \frac{r_{x}}{p_{x}}\right) \frac{r_{x}}{p_{x}}\right\rangle=\frac{3}{2} C,
$$

which means that $3 C=\left(1+\sigma_{q}^{2}\right)^{2}-4\left(1+\sigma_{q}^{2}\right)\left\langle q^{2}\right\rangle+3\left\langle q^{3}\right\rangle$, or

$$
C=\left\langle q^{3}\right\rangle-\left(1+\sigma_{q}^{2}\right)^{2}=\left\langle q^{3}\right\rangle-\left(\left\langle q^{2}\right\rangle\right)^{2} .
$$

Therefore, we have shown that the second derivative is

$$
\left.\frac{d^{2} \sigma_{W}}{d^{2}\langle W\rangle}\right|_{\gamma=\gamma_{c}}=\frac{C}{\gamma_{c}^{5}}
$$

Note that the positivity of second derivative is guaranteed in the general. Indeed using CauchySchwarz, $\left\langle q^{2}\right\rangle^{2} \leq\left\langle q^{3}\right\rangle$, which shows that $C \geq 0$, with equality iff $r_{x}^{1 / 2} \propto \frac{r_{x}^{3 / 2}}{p_{x}}$, i.e. when $p_{x}=r_{x}$.

In the end, we obtain the following second order approximation of the Pareto border near the null strategy

$$
\sigma_{W} \sim \frac{1}{\gamma_{c}}\langle W\rangle+\frac{C}{2 \gamma_{c}^{5}}\langle W\rangle^{2}
$$

In the particular case of two horses, it is straightforward to check that the expression of $\gamma_{c}$ given in Eq. B9 and that of the second derivative in Eq. B13 are recovered from Eq. B9 and Eq. D14 because of the following relations :

$$
\sigma_{q}^{2}=\frac{r^{2}}{p}+\frac{(1-r)^{2}}{1-p}-1=\frac{(r-p)^{2}}{\sigma^{2}},
$$

and

$$
\begin{aligned}
C & =-\sigma_{q}^{4}+\left\langle(q-1)^{3}\right\rangle+\sigma_{q}^{2}=-\frac{(p-r)^{4}}{\sigma^{4}}+\frac{(p-r)^{3}}{\sigma^{4}}(2 p-1)+\frac{(p-r)^{2}}{\sigma^{2}} \\
& =\frac{(p-r)^{2}}{\sigma^{4}}\left\{-(p-r)^{2}+(p-r)(2 p-1)+p(1-p)\right\} \\
& =\frac{(p-r)^{2}}{\sigma^{4}} r(1-r),
\end{aligned}
$$

therefore

$$
\frac{C}{\gamma_{c}^{5}}=\frac{r(1-r)}{\gamma_{c}^{3} \sigma^{2}}
$$

as expected. 


\section{Appendix E: General mathematical results for the shape of the border}

We prove in this section the general results stated p.4 and 5 of Main text.

\section{1. Large negative values of growth rate.}

Let $\emptyset \subsetneq X^{\prime} \subsetneq X$. Assume $b_{x} \rightarrow 0$ when $x \in X^{\prime}$, while all other parameters $\left(b_{x}\right)_{x \notin X^{\prime}}$ are kept fixed. Eq. (D5),

$$
\ln \left(\frac{b_{x}}{r_{x}}\right)+\mu \frac{b_{x}}{p_{x}}=m+\mu \quad \text { independent from } x
$$

implies that $\ln \left(b_{x} / r_{x}\right) \sim m+\mu$ independently of $x$ for all $x \in X^{\prime}$; thus the speed of convergence of $b_{x}$ to 0 for $x \in X^{\prime}$ may be characterized by a single parameter $\varepsilon \rightarrow 0^{+}$defined by $-1 / \varepsilon=\ln \left(b_{x^{\prime}} / r_{x^{\prime}}\right)$, $x^{\prime}$ being an arbitrary element of $X^{\prime}$; for all $x \in X^{\prime}, \ln \left(b_{x} / r_{x}\right) \sim-1 / \varepsilon$. Hence a first relation, where $0<P^{\prime}:=\sum_{x \in X^{\prime}} p_{x}<1$,

$$
\text { (1) } \quad m=\sum_{x} p_{x} \ln \left(b_{x} / r_{x}\right) \sim \sum_{x \in X^{\prime}} p_{x} \ln \left(b_{x} / r_{x}\right) \sim_{\varepsilon \rightarrow 0}-P^{\prime} / \varepsilon
$$

Now, $-1 / \varepsilon \sim m+\mu$, hence a second relation,

$$
\text { (2) } \quad m \sim_{\varepsilon \rightarrow 0} P^{\prime}(m+\mu) .
$$

From (1) and (2), we deduce $\mu \sim_{\varepsilon \rightarrow 0}-\left(1-P^{\prime}\right) / \varepsilon$.

Similarly,

$$
\sigma_{W}^{2}=\sum_{x} p_{x} \ln ^{2}\left(b_{x} / r_{x}\right)-m^{2} \sim P^{\prime} / \varepsilon^{2}-m^{2}=P^{\prime}\left(1-P^{\prime}\right) / \varepsilon^{2}
$$

whence a $P^{\prime}$-dependent asymptote for the front,

$$
\frac{\sigma_{W}}{|m|} \longrightarrow{ }_{\varepsilon \rightarrow 0} \sqrt{\frac{1-P^{\prime}}{P^{\prime}}} .
$$

This is in whole coherence with the two-horse case analyzed p.3. This gives a set of asymptotes with slopes $\left\{\sqrt{\frac{1-P^{\prime}\left(X^{\prime}\right)}{P^{\prime}\left(X^{\prime}\right)}}\right\}$, where $P^{\prime}\left(X^{\prime}\right):=\sum_{x \in X^{\prime}} p_{x}$, and $X^{\prime}$ ranges in the set of non trivial subsets of $X$. Note that the function $P^{\prime} \mapsto \sqrt{\frac{1-P^{\prime}}{P^{\prime}}}$ is decreasing, so the largest slope (highest asymptote) is $\sqrt{\frac{1-p_{\min }}{p_{\min }}}$, with $p_{\text {min }}:=\min _{x \in X} p_{x}$ (say, $p_{\text {min }}=p_{x^{*}}$ for some $x^{*} \in X$ ), while the smallest slope (lowest asymptote) is the inverse quantity, $\sqrt{\frac{p_{\min }}{1-p_{\min }}}$, obtained by choosing $X^{\prime}=X \backslash\left\{x^{*}\right\}$ - clearly, the worst possible strategy, since all bets are set on the worst horse - .

V. 2. Convexity of the lower front. The remainder of the section is devoted to the computation of the second derivative of the border $\frac{d^{2} \sigma_{W}}{d\langle W\rangle^{2}}$. We introduce the functional

$$
\tilde{J}_{m^{*}}(b ; \lambda, \mu):=\left\langle W^{2}\right\rangle+\lambda\left(\langle W\rangle-m^{*}\right)+2 \mu\left(\sum_{x} b_{x}-1\right),
$$

where $\lambda, \mu$ are Lagrange multipliers fixing $\langle W\rangle$ and implementing the bet normalization constraint. Conditions $\frac{\partial \tilde{J}_{m^{*}}}{\partial \lambda}=0, \frac{\partial \tilde{J}_{m^{*}}}{\partial \mu}=0$ fix the average growth rate $\langle W\rangle$ to the value $m^{*}$, and impose the constraint $\sum_{x} b_{x}=1$. Conditions $\frac{\partial \tilde{J}_{m^{*}}}{\partial b_{x}}=0$ then give the local extrema of $\sigma_{W}$ for fixed $\langle W\rangle$ since $\sigma_{W}^{2}=\left\langle W^{2}\right\rangle-\left(m^{*}\right)^{2}$. Depending on the eigenvalues of the Hessian $D^{2} \tilde{J}_{m^{*}}$, one may in principle select local maxima or minma; in practice this is however complicated due to the constraints. 
Theorem 1 Let us introduce the vectors

$$
a_{x}:=\frac{1}{2}\left(\nabla^{2} \tilde{J}_{m}\right)_{x x}=p_{x}\left(\frac{r_{x}}{b_{x}}\right)^{2}\left(1-\frac{\lambda}{2}-\ln \left(b_{x} / r_{x}\right)\right)
$$

and $u_{x}=p_{x} \frac{r_{x}}{b_{x}}$ and $v_{x}=2 r_{x}$ so that $a_{x}=\frac{u_{x}}{p_{x}}\left(u_{x}+\frac{\mu}{2} v_{x}\right)$. Then,

$$
\begin{aligned}
& \frac{d^{2} \sigma_{W}}{d\langle W\rangle^{2}}=\frac{4 \mu}{\sigma_{W}^{2} \operatorname{det}_{a}^{2}(u ; v)} \times \\
& \quad \times\left\{\frac{\sigma_{W}^{2}}{\mu^{2}}\left[\left(1-\frac{1}{2}(u, v)_{a}\right)-\frac{\mu}{4} \operatorname{det}_{a}^{2}(u ; v)\right]-\frac{\mu}{4} \operatorname{det}_{a}^{2}(u ; v)\right\}
\end{aligned}
$$

where $(\cdot, \cdot)_{a}, \operatorname{det}_{a}^{2}(\cdot, \cdot)$ refer to the (not necessarily positive-definite) "pseudo-metric" $g_{x y}=$ $a_{x}^{-1} \delta_{x, y}$ on $\mathbb{R}^{|X|}$, namely,

$$
(u, v)_{a}=\sum_{x} a_{x}^{-1} u_{x} v_{x}, \quad|u|_{a}^{2}=(u, u)_{a},|v|_{a}^{2}=(v, v)_{a}, \quad \operatorname{det}_{a}^{2}(u, v)=|u|_{a}^{2}|v|_{a}^{2}-(u, v)_{a}^{2}
$$

The above analytical formula is implicit, since $(b, \lambda, \mu)$ are functions of $\langle W\rangle$. Furthermore, it implies the following result:

Theorem 2 (i) The part of the lower front (see section 3)-i.e. of the variance-minimizing curve - with $\langle W\rangle>0$ is strictly convex, i.e. $\frac{d^{2} \sigma_{W}}{d m^{2}}>0$.

(ii) In some neighborhood of the null strategy on the left lower front defined by $\langle W\rangle<0$, and in some neighborhood of Kelly's strategy on the upper front, the front is concave, i.e. satisfies $\frac{d^{2} \sigma_{W}}{d m^{2}}<0$

\section{2. 1. Proof of Theorem 1}

Let us start from $\left(b=\left(b_{x}\right)_{x \in X}=b^{*}, \lambda=\lambda^{*}, \mu=\mu^{*} ; m=m^{*}\right)$ such that $f(b, \lambda, \mu ; m) \equiv$ $D \tilde{J}_{m^{*}}=0$. The gradient of $f$ w.r. to $(b, \lambda, \mu)$, which is the Hessian $D^{2} \tilde{J}_{m}$, is (as proved below) invertible. The implicit function theorem then implies that the locus $\{f=0\}$ is given locally around $\left(b^{*}, \lambda^{*}, \mu^{*} ; m^{*}\right)$ by functions $b=b(m), \lambda=\lambda(m), \mu=\mu(m)$ such that

$$
\frac{d b_{x}}{d m}=-\left((\tilde{\nabla} f)^{-1} \frac{\partial f}{\partial m}\right)_{x}, \quad \frac{d \lambda}{d m}=-\left((\tilde{\nabla} f)^{-1} \frac{\partial f}{\partial m}\right)_{\lambda}, \quad \frac{d \mu}{d m}=-\left((\tilde{\nabla} f)^{-1} \frac{\partial f}{\partial m}\right)_{\mu}
$$

In subsequent computations, we use rescaled variables $\varepsilon_{x}=\frac{b_{x}-b_{x}^{*}}{r_{x}}$ instead of $b$. The notation $(. .)_{x}$ denotes the component along $\varepsilon=\left(\varepsilon_{x}\right)_{x \in X}$ of the gradient. The condition $\sum_{x} \frac{b_{x}}{r_{x}} \nabla \tilde{J}_{m}=0$ yields $-\frac{\lambda}{2}=m^{*}+\mu$, which implies in turn the equations

$$
\ln \left(b_{x} / r_{x}\right)+\mu\left(\frac{b_{x}}{p_{x}}-1\right)=m,
$$

which are equivalent to (6) (see Main text) if one sets $\mu=\sigma_{W} / \gamma$.

A. 1. Computation of the inverse of the Hessian.

$$
\tilde{\nabla} f=\left(\begin{array}{c|cc}
\nabla^{2} \tilde{J}_{m} & \partial_{\lambda} \nabla \tilde{J} & \partial_{\mu} \nabla \tilde{J} \\
\hline\left(\partial_{\lambda} \nabla \tilde{J}\right)^{t} & \partial_{\lambda}^{2} \tilde{J}_{m} & \partial_{\lambda \mu}^{2} \tilde{J}_{m} \\
\left(\partial_{\mu} \nabla \tilde{J}\right)^{t} & \partial_{\lambda \mu}^{2} \tilde{J}_{m} & \partial_{\mu}^{2} \tilde{J}_{m}
\end{array}\right)=\left(\begin{array}{c|c}
A & B \\
\hline B^{t} & 0
\end{array}\right)
$$

where

$$
A=\nabla^{2} \tilde{J}_{m}=\left(\nabla_{x y}^{2} \tilde{J}_{m}\right)_{x y}, \quad B=\left(\partial_{\lambda} \nabla \tilde{J} \quad \partial_{\mu} \nabla \tilde{J}\right)
$$


are $|X| \times|X|$, resp. $|X| \times 2$ matrices, and $A=A^{t}$ is symmetric. The inverse of the matrix $\tilde{\nabla} f$ is, as follows from a simple computation,

$$
(\tilde{\nabla} f)^{-1}=\left(\begin{array}{c|c}
A^{-1}\left(I-B\left(A^{-1} B C^{-1}\right)^{t}\right) & A^{-1} B C^{-1} \\
\hline\left(\left(A^{-1}\right) B C^{-1}\right)^{t} & -C^{-1}
\end{array}\right)
$$

where $C=B^{t} A^{-1} B$ is a symmetric $2 \times 2$ matrix. Some elementary algebra yields in block form

$$
(\tilde{\nabla} f)^{-1}=\frac{1}{\operatorname{det}_{a}^{2}(u ; v)}\left(\begin{array}{c|c|c|}
\frac{1}{2}\left(\operatorname{det}_{a}^{2}(u ; v) \operatorname{diag}\left(a^{-1}\right)-Z\right) & |v|_{a}^{2} \overrightarrow{a^{-1} u}-(u, v)_{a} \overrightarrow{a^{-1} v}|u|_{a}^{2} \overrightarrow{a^{-1} v}-(u, v)_{a} \overrightarrow{a^{-1} u} \\
|v|_{a}^{2}\left(a^{-1} u\right)^{t}-(u, v)_{a}\left(a^{-1} v\right)^{t} & -2|v|_{a}^{2} & 2(u, v)_{a} \\
|u|_{a}^{2}\left(a^{-1} v\right)^{t}-(u, v)_{a}\left(a^{-1} u\right)^{t} & 2(u, v)_{a} & -2|u|_{a}^{2}
\end{array}\right)
$$

Note that $u_{x} \sim a_{x} \sim p_{x}$ in the neighborhood of the null strategy, so that (rescaling vectors $c, d \in \mathbb{R}^{|X|}$ by the probability weights $\left.p_{x},(p c)_{x}:=p_{x} c_{x},(p d)_{x}:=p_{x} d_{x}\right)(p c, p d)_{a} \sim \sum_{x} p_{x} c_{x} d_{x}$ boils down to the usual $L^{2}$-metric weighted by $p$ in that limit. In the equation above, we have denoted $Z$ the following symmetric $|X| \times|X|$-matrix,

$$
Z:=|v|_{a}^{2}\left(a^{-1} u \otimes a^{-1} u\right)+|u|_{a}^{2}\left(a^{-1} v \otimes a^{-1} v\right)-(u, v)_{a}\left\{\left(a^{-1} u \otimes a^{-1} v\right)+\left(a^{-1} v \otimes a^{-1} u\right)\right\}
$$

Then $\overrightarrow{a^{-1} u}$ is a vector in $\mathbb{R}^{|X|},\left(a^{-1} u\right)_{x}=a_{x}^{-1} u_{x}$, with transpose $\left(a^{-1} u\right)^{t}$.

A. 2. We easily derive from the inverse Hessian formula the first derivative of the Pareto border. We find first

$$
\frac{1}{2} \frac{d \sigma_{W}^{2}}{d m}=\frac{1}{2} \frac{d\left\langle W^{2}\right\rangle}{d m}-m=\frac{1}{2} \sum_{x} \frac{d \varepsilon_{x}}{d m} \frac{\partial\left\langle W^{2}\right\rangle}{\partial \varepsilon_{x}}-m
$$

since $\left\langle W^{2}\right\rangle$ is independent of $\lambda, \mu$ and does not depend explicitly on $m$

$$
=\sum_{x} p_{x} \frac{r_{x}}{b_{x}} \ln \left(b_{x} / r_{x}\right)\left(-(\tilde{\nabla} f)^{-1} \frac{\partial f}{\partial m}\right)_{x}-m=\sum_{x} p_{x} \frac{r_{x}}{b_{x}} \ln \left(b_{x} / r_{x}\right)\left((\tilde{\nabla} f)^{-1}\left(\begin{array}{l}
0 \\
1 \\
0
\end{array}\right)\right)_{x}-m .
$$

Then

$$
\begin{aligned}
& \frac{1}{2} \frac{d \sigma_{W}^{2}}{d m}=-m+\sum_{x} p_{x} \frac{r_{x}}{b_{x}} \ln \left(b_{x} / r_{x}\right)\left((\tilde{\nabla} f)^{-1}\left(\begin{array}{c}
0 \\
1 \\
0
\end{array}\right)\right)_{x} \\
& =-m+\frac{1}{\operatorname{det}_{a}^{2}(u ; v)} \sum_{x}\left(m u_{x}-\frac{\mu}{2}\left(v_{x}-2 u_{x}\right)\right)\left(|v|_{a}^{2} \frac{u_{x}}{a_{x}}-(u, v)_{a} \frac{v_{x}}{a_{x}}\right) \\
& =\frac{1}{\operatorname{det}_{a}^{2}(u ; v)} \mu\left(|v|_{a}^{2} \sum_{x} \frac{u_{x}}{a_{x}}-\left(2-\frac{\mu}{2}|v|_{a}^{2}\right) \sum_{x} \frac{v_{x}}{a_{x}}\right) \\
& =\mu
\end{aligned}
$$

The result is coherent with the slope formula $d \sigma_{W} / d\langle W\rangle=1 / \gamma$, if one sets $\mu=\sigma_{W} / \gamma$.

A. 3. Exploration of the front: a possible application of the inverse Hessian formula. Formula (E8), completed by the expression E13 for $(\tilde{\nabla} f)^{-1}$, allows a numerical exploration of the front starting from an arbitrary point. This provides an elementary alternative to the numerical exploration process described in Main Text.

B. 1. Second derivative. Preliminary results. We must differentiate once more to obtain $d^{2} \sigma_{W}^{2} / d\langle W\rangle^{2}=d^{2} \sigma_{W}^{2} / d m^{2}$. This is straightforward in principle using the explicit expression for $(\tilde{\nabla} f)^{-1}$, but in practice, computations are rather involved. 
Differentiating E15) once again, one finds

$$
1+\frac{1}{2} \frac{d^{2}\left(\sigma_{W}^{2}\right)}{d m^{2}} \equiv D_{1}^{2}+D_{2}^{2}+D_{3}^{2}
$$

with

$$
D_{1}^{2}:=\frac{1}{2} \sum_{x}\left(\frac{d \varepsilon_{x}}{d m}\right)^{2} \frac{\partial^{2}\left(\left\langle w^{2}\right\rangle\right)}{\partial \varepsilon_{x}^{2}}=\sum_{x} a_{x}^{0}\left((\tilde{\nabla} f)^{-1}\left(\begin{array}{l}
0 \\
1 \\
0
\end{array}\right)\right)_{x}^{2}
$$

where

$$
\begin{aligned}
a_{x}^{0}:=\left.\frac{1}{2} p_{x} \frac{d^{2}}{d \varepsilon^{2}} \ln ^{2}\left(\frac{b_{x}}{r_{x}}+\varepsilon\right)\right|_{\varepsilon=0}=p_{x}\left(\frac{r_{x}}{b_{x}}\right)^{2}\left(1-\ln \left(b_{x} / r_{x}\right)\right)=\frac{u_{x}}{p_{x}}\left\{(1-m-\mu) u_{x}+\frac{\mu}{2} v_{x}\right\} ; \\
D_{2}^{2}:=\frac{1}{2} \frac{d \lambda}{d m} \sum_{x} \frac{\partial\langle w\rangle^{2}}{\partial \varepsilon_{x}} \frac{\partial}{\partial \lambda}\left(\frac{d \varepsilon_{x}}{d m}\right) \\
=\left((\tilde{\nabla} f)^{-1}\left(\begin{array}{c}
0 \\
1 \\
0
\end{array}\right)\right)_{\lambda} \sum_{x} p_{x} \frac{r_{x}}{b_{x}} \ln \left(\frac{b_{x}}{r_{x}}\right) \frac{\partial}{\partial \lambda}\left((\tilde{\nabla} f)^{-1}\left(\begin{array}{c}
0 \\
1 \\
0
\end{array}\right)\right)_{x} \\
D_{3}^{2}:=\frac{1}{2} \sum_{x, y} \frac{\partial\left\langle w^{2}\right\rangle}{\partial \varepsilon_{x}} \frac{d \varepsilon_{y}}{d m} \frac{\partial}{\partial \varepsilon_{y}}\left(\frac{d \varepsilon_{x}}{d m}\right) \\
=\sum_{x} p_{x} \frac{r_{x}}{b_{x}} \ln \left(\frac{b_{x}}{r_{x}}\right) \sum_{y}\left((\tilde{\nabla} f)^{-1}\left(\begin{array}{c}
0 \\
1 \\
0
\end{array}\right)\right)_{y} \frac{\partial}{\partial \varepsilon_{y}}\left((\tilde{\nabla} f)^{-1}\left(\begin{array}{c}
0 \\
1 \\
0
\end{array}\right)\right)_{x} .
\end{aligned}
$$

Upon computing these quantities for $(\varepsilon, \lambda, \mu ; m)=(0, \lambda(m), \mu(m) ; m)$, we can use D5 and replace in the sum $\ln \left(b_{x} / r_{x}\right)$ by $m-\mu\left(\frac{b_{x}}{p_{x}}-1\right)$.

The only coefficients of the inverse Hessian that we need are

$$
\left((\tilde{\nabla} f)^{-1}\left(\begin{array}{c}
0 \\
1 \\
0
\end{array}\right)\right)_{x}=\frac{1}{\operatorname{det}_{a}^{2}(u ; v)}\left(|v|_{a}^{2} \frac{u_{x}}{a_{x}}-(u, v)_{a} \frac{v_{x}}{a_{x}}\right) .
$$

and

$$
\left((\tilde{\nabla} f)^{-1}\left(\begin{array}{c}
0 \\
1 \\
0
\end{array}\right)\right)_{\lambda}=-\frac{2|v|_{a}^{2}}{\operatorname{det}_{a}^{2}(u ; v)}
$$

B. 2. Remarkable identities Computations for the second derivative involve the following sums, $I_{i, j}=\sum_{x} a_{x}^{-1} u_{x}^{i} v_{x}^{j}(i+j=2), I_{i, j}=\sum_{x} a_{x}^{-1} \frac{u_{x}^{i} v_{x}^{j}}{p_{x} a_{x}}(i+j=4), I_{i, j}=\sum_{x} a_{x}^{-1} \frac{u_{x}^{i} v_{x}^{j}}{\left(p_{x} a_{x}\right)^{2}}(i+j=6)$. The explicit expressions for $a$ in terms of $u, v$, together with the normalization condition $\sum_{x} p_{x}=$ $\sum_{x} b_{x}=1$, yield a set of remarkable identities giving a triangular structure,

$$
I_{4,0}+\frac{\mu}{2} I_{3,1}=|u|_{a}^{2}, \quad \frac{\mu}{2} I_{2,2}+I_{3,1}=(u, v)_{a}, \quad I_{2,2}+\frac{\mu}{2} I_{1,3}=|v|_{a}^{2}
$$

and

$$
I_{6,0}+\frac{\mu}{2} I_{5,1}=I_{4,0}, \quad I_{5,1}+\frac{\mu}{2} I_{4,2}=I_{3,1},
$$




$$
I_{4,2}+\frac{\mu}{2} I_{3,3}=I_{2,2}, \quad I_{3,3}+\frac{\mu}{2} I_{2,4}=I_{1,3}
$$

The more elementary identities

$$
|u|_{a}^{2}+\frac{\mu}{2}(u, v)_{a}=1, \quad \frac{\mu}{2}|v|_{a}^{2}+(u, v)_{a}=2
$$

can be proven similarly, from which

$$
\operatorname{det}_{a}^{2}(u ; v)=\left(1-\frac{\mu}{2}(u, v)_{a}\right) \frac{2}{\mu}\left(2-(u, v)_{a}\right)-(u, v)_{a}^{2}=\frac{2}{\mu}\left(2-(\mu+1)(u, v)_{a}\right) .
$$

They imply after tedious computations:

$$
\begin{aligned}
D_{1}^{2}= & \frac{1}{\operatorname{det}_{a}^{4}(u ; v)}\left\{(1-m-\mu)\left[-\frac{8}{\mu} I_{3,1}+\frac{4}{\mu^{2}}\left(4-(4+2 \mu)(u, v)_{a}+(1+2 \mu)(u, v)_{a}^{2}\right)\right]\right. \\
+ & \left.\frac{\mu}{2}\left[-\frac{8}{\mu} I_{2,2}-\frac{8}{\mu^{2}}(u, v)_{a}\left((u, v)_{a}-2\right)\right]\right\} .
\end{aligned}
$$

B. 3. (computation of the second term $\left.D_{2}^{2}\right)$. We prove that $D_{2}^{2}=0$. Let $\tilde{M}(\lambda):=\tilde{\nabla} f=$ $\left(\begin{array}{c|c}M(\lambda) & \text { Cst } \\ \hline \text { Cst } & \text { Cst }\end{array}\right)$, then

$$
\begin{aligned}
& \frac{\partial}{\partial \lambda}\left((\tilde{\nabla} f)^{-1}\left(\begin{array}{l}
0 \\
1 \\
0
\end{array}\right)\right)_{x}=-\left(\tilde{M}(\lambda) \frac{d \tilde{M}}{d \lambda} \tilde{M}^{-1}(\lambda)\left(\begin{array}{l}
0 \\
1 \\
0
\end{array}\right)\right)_{x} \\
& =\left((\tilde{\nabla} f)^{-1}\left(\begin{array}{c|c}
\operatorname{diag}\left(p_{x}\left(\frac{r_{x}}{b_{x}}\right)^{2}\right) & 0 \\
\hline 0 & 0
\end{array}\right)(\tilde{\nabla} f)^{-1}\left(\begin{array}{l}
0 \\
1 \\
0
\end{array}\right)\right)_{x} \\
& =\frac{1}{\operatorname{det}_{a}^{2}(u ; v)}\left((\tilde{\nabla} f)^{-1}\left(\begin{array}{c|c}
\operatorname{diag}\left(\frac{u_{x}^{2}}{p_{x}}\right) & 0 \\
\hline 0 & 0
\end{array}\right)\left(\begin{array}{c}
|v|_{a}^{2} \frac{u_{x}}{a_{x}}-(u, v)_{a} \frac{v_{x}}{a_{x}} \\
-2|v|_{a}^{2} \\
2(u, v)_{a}
\end{array}\right)\right)_{x} \\
& =\frac{1}{\operatorname{det}_{a}^{2}(u ; v)}\left((\tilde{\nabla} f)^{-1}\left(\begin{array}{c}
w_{x} \\
0 \\
0
\end{array}\right)\right)_{x}=\frac{1}{2 \operatorname{det}_{a}^{4}(u ; v)}\left(\left(\operatorname{det}_{a}^{2}(u ; v) \operatorname{diag}\left(a^{-1}\right)-Z\right) w\right)_{x},
\end{aligned}
$$

where using the above remarkable identities,

$w_{x}:=\frac{u_{x}^{2}}{p_{x}}\left(|v|_{a}^{2} \frac{u_{x}}{a_{x}}-(u, v)_{a} \frac{v_{x}}{a_{x}}\right)=\frac{u_{x}^{2}}{p_{x}}\left(\frac{4}{\mu} \frac{u_{x}}{a_{x}}-(u, v)_{a} \frac{v_{x}+\frac{2}{\mu} u_{x}}{a_{x}}\right)=\frac{1}{p_{x} a_{x}}\left\{\frac{4}{\mu} u_{x}^{3}-(u, v)_{a}\left(\frac{2}{\mu} u_{x}^{3}+u_{x}^{2} v_{x}\right)\right\}$.

Computation of $(Z w)$-term. $Z \equiv Z_{1}+Z_{2}-\left(Z_{3}+Z_{3}^{\text {sym }}\right)$, with $Z_{1}:=|v|_{a}^{2}\left(a^{-1} u \otimes a^{-1} u\right), Z_{2}:=$ $|u|_{a}^{2}\left(a^{-1} v \otimes a^{-1} v\right), Z_{3}:=(u, v)_{a}\left(a^{-1} u \otimes a^{-1} v\right), Z_{3}^{s y m}:=(u, v)_{a}\left(a^{-1} v \otimes a^{-1} u\right)$. Using the remarkable identities, we get

$$
\begin{aligned}
& \left(Z_{1} w\right)_{x}=|v|_{a}^{2} \frac{u_{x}}{a_{x}}\left\{\left(\frac{4}{\mu}-\frac{2}{\mu}(u, v)_{a}\right) I_{4,0}-(u, v)_{a} I_{3,1}\right\}=|v|_{a}^{2} \frac{u_{x}}{a_{x}}\left\{\frac{2}{\mu}|u|_{a}^{2}\left(2-(u, v)_{a}\right)-2 I_{3,1}\right\} \\
& \left(Z_{3} w\right)_{x}=(u, v)_{a} \frac{u_{x}}{a_{x}}\left\{\frac{2}{\mu}\left(2-(u, v)_{a}\right) I_{3,1}-(u, v)_{a} I_{2,2}\right\}=(u, v)_{a} \frac{u_{x}}{a_{x}} \frac{2}{\mu}\left\{2 I_{3,1}-(u, v)_{a}^{2}\right\} \\
& \left(\left(Z_{1}-Z_{3}\right) w\right)_{x}=\frac{u_{x}}{a_{x}}\left[I_{3,1}\left\{-2|v|_{a}^{2}-\frac{4}{\mu}(u, v)_{a}\right\}+\left(\frac{2}{\mu}\right)^{2}\left(1-\frac{\mu}{2}(u, v)_{a}\right)\left(2-(u, v)_{a}\right)^{2}+\frac{2}{\mu}(u, v)_{a}^{3}\right] \\
& =\frac{u_{x}}{a_{x}}\left\{-\frac{8}{\mu} I_{3,1}+\frac{4}{\mu^{2}}\left[4-(4+2 \mu)(u, v)_{a}+(1+2 \mu)(u, v)_{a}^{2}\right]\right\}
\end{aligned}
$$


Similarly, $\quad\left(Z_{2} w\right)_{x}=|u|_{a}^{2} \frac{v_{x}}{a_{x}} \frac{2}{\mu}\left\{2 I_{3,1}-(u, v)_{a}^{2}\right\} \quad$ (compare with $\left.\operatorname{E32}\right) ; \quad\left(Z_{3}^{\text {sym }} w\right)_{x}=$ $(u, v)_{a} \frac{v_{x}}{a_{x}}\left\{\frac{2}{\mu}|u|_{a}^{2}\left(2-(u, v)_{a}\right)-2 I_{3,1}\right\} \quad$ (compare with $\quad$ E31) $) ; \quad\left(\left(Z_{2}-Z_{3}^{\text {sym }}\right) w\right)_{x}=$ $\frac{v_{x}}{a_{x}}\left\{\frac{4}{\mu} I_{3,1}-\frac{4}{\mu}|u|_{a}^{2}(u, v)_{a}\right\}$. The contribution of the term in $\left(Z_{1}-Z_{3}\right) w$ to $C_{2}:=$ $2 \operatorname{det}_{a}^{4}(u ; v) \sum_{x} p_{x} \frac{r_{x}}{b_{x}} \ln \left(\frac{b_{x}}{r_{x}}\right) \frac{\partial}{\partial \lambda}\left((\tilde{\nabla} f)^{-1}\left(\begin{array}{l}0 \\ 1 \\ 0\end{array}\right)\right)_{x}$ is

$$
\begin{aligned}
& \sum_{x}\left(m u_{x}-\frac{\mu}{2}\left(v_{x}-2 u_{x}\right)\right) \frac{u_{x}}{a_{x}}\left\{-\frac{8}{\mu} I_{3,1}+\frac{4}{\mu^{2}}\left[4-(4+2 \mu)(u, v)_{a}+(1+2 \mu)(u, v)_{a}^{2}\right]\right\} \\
& =(m+\mu)|u|_{a}^{2}\left\{-\frac{8}{\mu} I_{3,1}+\frac{4}{\mu^{2}}\left[4-(4+2 \mu)(u, v)_{a}+(1+2 \mu)(u, v)_{a}^{2}\right]\right\} \\
& \quad-\frac{\mu}{2}(u, v)_{a}\left\{-\frac{8}{\mu} I_{3,1}+\frac{4}{\mu^{2}}\left[4-(4+2 \mu)(u, v)_{a}+(1+2 \mu)(u, v)_{a}^{2}\right]\right\}
\end{aligned}
$$

Similarly, the contribution of the term in $\left(Z_{2}-Z_{3, \text { sym }}\right) w$ to $C_{2}$ is

$$
\begin{aligned}
& \sum_{x}\left(m u_{x}-\frac{\mu}{2}\left(v_{x}-2 u_{x}\right)\right) \frac{v_{x}}{a_{x}}\left\{\frac{4}{\mu} I_{3,1}-\frac{4}{\mu}|u|_{a}^{2}(u, v)_{a}\right\} \\
& =(m+\mu)(u, v)_{a}\left\{\frac{4}{\mu} I_{3,1}-\frac{4}{\mu}|u|_{a}^{2}(u, v)_{a}\right\}-\frac{\mu}{2}|v|_{a}^{2}\left\{\frac{4}{\mu} I_{3,1}-\frac{4}{\mu}|u|_{a}^{2}(u, v)_{a}\right\}
\end{aligned}
$$

Contribution of the diagonal term. Finally, the contribution of the term $\operatorname{in}_{\operatorname{det}_{a}^{2}}^{2}(u ; v) \frac{w_{x}}{a_{x}}$ to $C_{2}$ is

$$
\begin{aligned}
& \operatorname{det}_{a}^{2}(u ; v) \sum_{x}\left(m u_{x}-\frac{\mu}{2}\left(v_{x}-2 u_{x}\right)\right) \frac{1}{p_{x} a_{x}^{2}}\left\{\frac{4}{\mu} u_{x}^{3}-(u, v)_{a}\left(\frac{2}{\mu} u_{x}^{3}+u_{x}^{2} v_{x}\right)\right\} \\
& =\operatorname{det}_{a}^{2}(u ; v)\left\{(m+\mu)\left[\frac{4}{\mu}\left(|u|_{a}^{2}-\frac{\mu}{2} I_{3,1}\right)-\frac{2}{\mu}|u|_{a}^{2}(u, v)_{a}\right]\right. \\
& \left.\quad-\frac{\mu}{2}\left[\frac{4}{\mu} I_{3,1}-\frac{2}{\mu}(u, v)_{a}^{2}\right]\right\}
\end{aligned}
$$

Adding up the three terms E34, E35 and E36, and using formula E26 for $\operatorname{det}_{a}^{2}(u ; v)$, one gets zero after some extra work.

The partial derivative of $(\tilde{\nabla} f)^{-1}$ w.r. $\quad$ to $\varepsilon_{y}$ is computed as (compare with B.3.) $-(\tilde{\nabla} f)^{-1} \frac{\partial \tilde{\nabla} f}{\partial \varepsilon_{y}}(\tilde{\nabla} f)^{-1}$. Now,

$$
\left(\frac{\partial}{\partial \varepsilon_{y}} A\right)_{x x}=\left.\frac{\partial}{\partial \varepsilon_{y}}\left[\frac{2 p_{x}}{\left(\frac{b_{x}}{r_{x}}+\varepsilon_{x}\right)^{2}}\left(1-\frac{\lambda}{2}-\ln \left(\frac{b_{x}}{r_{x}}+\varepsilon_{x}\right)\right)\right]\right|_{\varepsilon=0}=-2 \delta_{x, y} p_{y}\left(\frac{r_{y}}{b_{y}}\right)^{3}\left(3-\lambda-2 \ln \left(\frac{b_{y}}{r_{y}}\right)\right)
$$

Writing $B=\left(B_{\cdot, 1} B_{\cdot, 2}\right)$, with $\left(B_{\cdot, 1}\right)_{x}=\left(\frac{p_{x}}{\frac{b_{x}}{r_{x}}+\varepsilon_{x}}\right),\left(B_{\cdot, 2}\right)=\left(2 r_{x}\right)_{x}$, we get $\nabla B_{\cdot, 2}=0$ and

$$
\left.\left(\frac{\partial}{\partial \varepsilon_{y}} B \cdot, 1\right)_{x}\right|_{\varepsilon=0}=-\delta_{x, y} p_{y}\left(\frac{r_{y}}{b_{y}}\right)^{2}
$$

All together, all coefficients of $\left.\frac{\partial}{\partial \varepsilon_{y}} \tilde{\nabla} f\right|_{\varepsilon=0}$ vanish, except three of them,

$$
\alpha_{y}:=\left(\frac{\partial}{\partial \varepsilon_{y}} \tilde{\nabla} f\right)_{y, y}=-2 p_{y}\left(\frac{r_{y}}{b_{y}}\right)^{3}\left(3-\lambda-2 \ln \left(\frac{b_{y}}{r_{y}}\right)\right)=-4\left(\frac{u_{y}}{p_{y}}\right)^{2}\left(\frac{3}{2} u_{y}+\frac{\mu}{2} v_{y}\right)
$$

and

$$
\beta_{y}:=\left(\frac{\partial}{\partial \varepsilon_{y}} \tilde{\nabla} f\right)_{\lambda, y}=\left(\frac{\partial}{\partial \varepsilon_{y}} \tilde{\nabla} f\right)_{y, \lambda}=-p_{y}\left(\frac{r_{y}}{b_{y}}\right)^{2}=-\frac{u_{y}^{2}}{p_{y}} .
$$

Then, successively, 
(i) the vector $w_{y}:=\left(\frac{\partial}{\partial \varepsilon_{y}} \tilde{\nabla} f\right)(\tilde{\nabla} f)^{-1}\left(\begin{array}{l}0 \\ 1 \\ 0\end{array}\right)$ has only two non-vanishing coefficients,

$$
\begin{gathered}
\left(w_{y}\right)_{y}:=\left(\left(\frac{\partial}{\partial \varepsilon_{y}} \tilde{\nabla} f\right)(\tilde{\nabla} f)^{-1}\left(\begin{array}{c}
0 \\
1 \\
0
\end{array}\right)\right)_{y} \equiv \frac{1}{\operatorname{det}_{a}^{2}(u ; v)} \gamma_{y}, \\
\gamma_{y}:=\alpha_{y}\left(|v|_{a}^{2} \frac{u_{y}}{a_{y}}-(u, v)_{a} \frac{v_{y}}{a_{y}}\right)+\beta_{y}\left(-2|v|_{a}^{2}\right)
\end{gathered}
$$

and

$$
\left(w_{y}\right)_{\lambda}:=\left(\left(\frac{\partial}{\partial \varepsilon_{y}} \tilde{\nabla} f\right)(\tilde{\nabla} f)^{-1}\left(\begin{array}{l}
0 \\
1 \\
0
\end{array}\right)\right)_{\lambda}=\frac{1}{\operatorname{det}_{a}^{2}(u ; v)} \beta_{y}\left(|v|_{a}^{2} \frac{u_{y}}{a_{y}}-(u, v)_{a} \frac{v_{y}}{a_{y}}\right) .
$$

(ii) (non-diagonal coefficient) if $x \neq y$, then

$$
\begin{aligned}
& -\left((\tilde{\nabla} f)^{-1}\left(\frac{\partial}{\partial \varepsilon_{y}} \tilde{\nabla} f\right)(\tilde{\nabla} f)^{-1}\left(\begin{array}{c}
0 \\
1 \\
0
\end{array}\right)\right)_{x} \\
& \quad=\frac{1}{\operatorname{det}_{a}^{4}(u ; v)}\left[\frac{1}{2} Z_{x, y} \gamma_{y}-\left(|v|_{a}^{2} \frac{u_{x}}{a_{x}}-(u, v)_{a} \frac{v_{x}}{a_{x}}\right) \beta_{y}\left(|v|_{a}^{2} \frac{u_{y}}{a_{y}}-(u, v)_{a} \frac{v_{y}}{a_{y}}\right)\right]
\end{aligned}
$$

(iii) (diagonal coefficient) letting $x=y$, then

$$
\begin{aligned}
& -\left((\tilde{\nabla} f)^{-1}\left(\frac{\partial}{\partial \varepsilon_{x}} \tilde{\nabla} f\right)(\tilde{\nabla} f)^{-1}\left(\begin{array}{c}
0 \\
1 \\
0
\end{array}\right)\right)_{x} \\
& \quad=\frac{1}{\operatorname{det}_{a}^{4}(u ; v)}\left[-\frac{1}{2}\left(\operatorname{det}_{a}^{2}(u ; v) a_{x}^{-1}-Z_{x, x}\right) \gamma_{x}-\left(|v|_{a}^{2} \frac{u_{x}}{a_{x}}-(u, v)_{a} \frac{v_{x}}{a_{x}}\right) \beta_{x}\left(|v|_{a}^{2} \frac{u_{x}}{a_{x}}-(u, v)_{a} \frac{v_{x}}{a_{x}}\right)\right]
\end{aligned}
$$

We now split accordingly the third line of $(\mathrm{E} 22)$,

$$
D_{3}^{2}:=\sum_{x} p_{x} \frac{r_{x}}{b_{x}} \ln \left(\frac{b_{x}}{r_{x}}\right) \sum_{y}\left((\tilde{\nabla} f)^{-1}\left(\begin{array}{l}
0 \\
1 \\
0
\end{array}\right)\right)_{y} \frac{\partial}{\partial \varepsilon_{y}}\left((\tilde{\nabla} f)^{-1}\left(\begin{array}{l}
0 \\
1 \\
0
\end{array}\right)\right)_{x} .
$$

into the sum of three contributions,

$$
D_{3}^{2} \equiv \frac{1}{\operatorname{det}_{a}^{6}(u ; v)}\left(D_{3, \beta}^{2}+D_{3, \gamma}^{2}+D_{3, \gamma, \text { diag }}^{2}\right) .
$$

Note that, now that we are located on the front, we can use (D5) and replace in the sum $p_{x} \frac{r_{x}}{b_{x}} \ln \left(b_{x} / r_{x}\right)$ by $m u_{x}-\frac{\mu}{2}\left(v_{x}-2 u_{x}\right)$ (as in A.2), while $\alpha_{y}, \beta_{y}$ are given by E39, (E40).

By tedious computations, we find :

$$
\begin{aligned}
& D_{3, \beta}^{2}=-\sum_{x, y}\left(m u_{x}-\frac{\mu}{2}\left(v_{x}-2 u_{x}\right)\right)\left(|v|_{a}^{2} \frac{u_{x}}{a_{x}}-(u, v)_{a} \frac{v_{x}}{a_{x}}\right) \beta_{y}\left(|v|_{a}^{2} \frac{u_{y}}{a_{y}}-(u, v)_{a} \frac{v_{y}}{a_{y}}\right)^{2} \\
& =\left\{\sum_{y} \frac{u_{y}^{2}}{p_{y}}\left(|v|_{a}^{2} \frac{u_{y}}{a_{y}}-(u, v)_{a} \frac{v_{y}}{a_{y}}\right)^{2}\right\}\left\{(m+\mu) \operatorname{det}_{a}^{2}(u ; v)\right\} \\
& =(m+\mu) \operatorname{det}_{a}^{2}(u ; v) \times \frac{4}{\mu^{2}}\left\{(1+2 \mu)(u, v)_{a}^{2}-2(2+\mu)(u, v)_{a}+4\right\}-\frac{8}{\mu} I_{3,1}
\end{aligned}
$$


Similar computations (which we choose to skip) yield $D_{3, \gamma}^{2}+D_{3, \gamma, \text { diag }}^{2}=0$. All together, we have found:

$$
1+\frac{1}{2} \frac{d^{2}\left(\sigma_{W}^{2}\right)}{d m^{2}}=\frac{1}{\operatorname{det}_{a}^{2}(u ; v)} \frac{2}{\mu}\left(2-(u, v)_{a}\right)
$$

B. 5. (final formula). Finally, from this last formula, we obtain

$$
\begin{aligned}
& \sigma_{W} \frac{d^{2} \sigma_{W}}{d m^{2}}=\frac{1}{2} \frac{d^{2}\left(\sigma_{W}^{2}\right)}{d m^{2}}-\frac{1}{\sigma_{W}^{2}}\left(\frac{1}{2} \frac{d \sigma_{W}^{2}}{d m}\right)^{2}=E 49-1-\frac{\mu^{2}}{\sigma_{W}^{2}} \\
& =\frac{4 / \mu}{\operatorname{det}_{a}^{2}(u ; v)}\left\{\left(1-\frac{1}{2}(u, v)_{a}\right)-\frac{\mu}{4} \operatorname{det}_{a}^{2}(u ; v)\left(1+\frac{\mu^{2}}{\sigma_{W}^{2}}\right)\right\} \\
& \equiv \frac{4 \mu}{\sigma_{W}^{2} \operatorname{det}_{a}^{2}(u ; v)} \times \mathcal{D}^{2},
\end{aligned}
$$

where

$$
\mathcal{D}^{2}:=\frac{\operatorname{det}_{a}^{2}(u ; v)}{4} \sigma_{W}^{3} \frac{d^{2} \sigma_{W}}{d m^{2}}=\frac{\sigma_{W}^{2}}{\mu^{2}}\left[\left(1-\frac{1}{2}(u, v)_{a}\right)-\frac{\mu}{4} \operatorname{det}_{a}^{2}(u ; v)\right]-\frac{\mu}{4} \operatorname{det}_{a}^{2}(u ; v)
$$

Ths implies, finally, Theorem 1

\section{2. 2. Proof of Theorem 2 ,}

1. General notations. For the proof, we first introduce new notations:

$$
s_{x}=\frac{p_{x}}{b_{x}},
$$

so that $u_{x}=r_{x} s_{x}$, and $\frac{u_{x}}{v_{x}}=\frac{u_{x}}{2 r_{x}}=\frac{s_{x}}{2}$; recall $\frac{1}{a_{x}}=\frac{p_{x}}{u_{x}\left(u_{x}+\frac{\mu}{2} v_{x}\right)}$, a formula which connects scalar products $(\cdot, \cdot)_{a}$ to standard averages $\langle\cdot\rangle$. Also, $\left\langle\frac{1}{s}\right\rangle=\sum_{x} b_{x}=1$. Finally, let

$$
L_{\mu}:=\frac{1}{\mu}\left(1-\left\langle\frac{1}{s+\mu}\right\rangle\right), \quad S:=\left\langle\frac{1}{s^{2}}\right\rangle=\left.\frac{d\left(\mu L_{\mu}\right)}{d \mu}\right|_{\mu=0}, \quad C:=\left\langle\frac{1}{s^{3}}\right\rangle=-\left.\frac{1}{2} \frac{d^{2}\left(\mu L_{\mu}\right)}{d \mu^{2}}\right|_{\mu=0}
$$

( $\mathrm{S}=$ square, $\mathrm{C}=$ cube). Then

$$
\begin{gathered}
\frac{\sigma_{W}^{2}}{\mu^{2}}=\frac{1}{\mu^{2}}\left(\left\langle W^{2}\right\rangle-m^{2}\right)=\frac{1}{\mu^{2}}\left\{\left\langle\left(m-\mu\left(\frac{b_{x}}{p_{x}}-1\right)\right)^{2}\right\rangle-m^{2}\right\} \\
=\left\langle\left(\frac{b_{x}}{p_{x}}-1\right)^{2}\right\rangle=\left\langle\frac{1}{s^{2}}\right\rangle-1=S-1 \\
1-\frac{1}{2}(u, v)_{a}=1-\left\langle\frac{1}{s+\mu}\right\rangle=\mu L_{\mu}, \\
1-\frac{1}{2}(1+\mu)(u, v)_{a}=1-(1+\mu)\left\langle\frac{1}{s+\mu}\right\rangle=\frac{\mu}{4} \operatorname{det}_{a}^{2}(u ; v)=\mu\left[(1+\mu) L_{\mu}-1\right],
\end{gathered}
$$

hence $\frac{d^{2} \sigma_{W}}{d m^{2}}$ has same sign as

$$
\begin{gathered}
\frac{1}{4} \operatorname{det}_{a}^{2}(u ; v) \frac{\mathcal{D}^{2}}{\mu}=\left\{(1+\mu) L_{\mu}-1\right\}\left\{(S-1) L_{\mu}-S\left((1+\mu) L_{\mu}-1\right)\right\} \\
=\left\{(1+\mu) L_{\mu}-1\right\}\left\{S-(1+S \mu) L_{\mu}\right\}
\end{gathered}
$$

2. Main Lemma. The proof of Theorem 2 rests on the following 
Lemma 1 1. Assume that $\mu>\max \left(-1, \max \left\{-s_{x}, x \in X\right\}\right)$ or

$\mu<\min \left(-1, \min \left\{-s_{x}, x \in X\right\}\right)$. Then $(1+\mu) L_{\mu}-1>0$.

2. Assume that $\mu>\max \left(-1 / S, \max \left\{-s_{x}, x \in X\right\}\right)$ or

$\mu<\min \left(-1 / S, \min \left\{-s_{x}, x \in X\right\}\right)$. Then $\left\langle\frac{1+S \mu}{s+\mu}\right\rangle>1$.

The conditions of the Lemma hold true in particular (i) if $\mu \geq 0$; (ii) if $-1 \ll \mu<0$ (including in particular the case of the part of the lower front with $\langle w\rangle<0$, as we shall see); (iii) if $\mu \ll-1$ (including in particular a neighborhood of Kelly's strategy - where $\mu=-\infty$ - on the upper front).

\section{Proof.}

1. We first note that $\mu\left((1+\mu) L_{\mu}-1\right)=(1+\mu)\left(1-\left\langle\frac{1}{s+\mu}\right\rangle\right)-\mu=1-\left\langle\frac{\mu+1}{\mu+s}\right\rangle$. Thus we need to prove that $x(\mu):=\left\langle\frac{\mu+1}{\mu+s}\right\rangle<1$, resp. $>1$, for $\mu>0$, resp. $\mu<0$. Note first that $x(0)=\left\langle\frac{1}{s}\right\rangle=1$.

Let $y(\mu):=\left\langle(1-s) \frac{\mu+1}{\mu+s}\right\rangle$. Mind that $y$ is considered here as a function of $\mu$ for $b=\left(b_{x}\right)$ fixed. Then

$$
\frac{d y}{d \mu}=-\left\langle\frac{(1-s)^{2}}{(\mu+s)^{2}}\right\rangle<0
$$

Since $y(0)=\left\langle\frac{1}{s}\right\rangle-1=0$, this means that $y(\mu)$ has opposite sign w.r. to $\mu$. When $\mu>0$, we get $y(\mu)<0$, or equivalently,

$$
x(\mu)<(1+\mu)\left\langle\frac{s}{s+\mu}\right\rangle=(1+\mu)\left(1-\left\langle\frac{\mu}{s+\mu}\right\rangle\right)=1+\mu-\mu x(\mu)
$$

whence $x(\mu)<1$. When $\max \left(-1, \max \left\{-s_{x}, x \in X\right\}\right)<\mu<0$, we get $y(\mu)>0$, from which $x(\mu)>1$.

Consider now the case $\mu<\min \left(-1, \min \left\{-s_{x}, x \in X\right\}\right)$. Let $\mu \rightarrow-\infty$, then $y(-\infty)=$ $1-\langle s\rangle=1-\left\langle\left(\frac{1}{s}\right)^{-1}\right\rangle \leq 1-\left\langle\frac{1}{s}\right\rangle^{-1}=0$ by Jensen's inequality (which is coherent with the previous analysis in the neighborhood of Kelly's strategy, since $y(-\infty)=y(+\infty)<0)$. Then E59) still holds, so $y(\mu)<0$, or equivalently, $x(\mu)<1+\mu-\mu x(\mu)$ (see (E60), and then (since $\mu<-1$ ) $x(\mu)>1$.

2. We let this time $x(\mu):=\left\langle\frac{S \mu+1}{\mu+s}\right\rangle$ and $y(\mu):=\left\langle\left(S-\frac{1}{s}\right) \frac{S \mu+1}{\mu+s}\right\rangle$. As in the previous point, only $\mu$ is varied. Then

$$
\frac{d y}{d \mu}=\left\langle\frac{(S s-1)\left(S-\frac{1}{s}\right)}{(\mu+s)^{2}}\right\rangle>0
$$

and $y(0)=0$, hence $y(\mu)$ has same sign as $\mu$. When $\mu>0$, we get $y(\mu)>0$, or equivalently,

$$
\begin{aligned}
& S x(\mu)>\left\langle\frac{S \mu+1}{s(\mu+s)}\right\rangle \\
& =\left\langle\frac{1}{s} \frac{S(\mu+s)+1-S s}{\mu+s}\right\rangle=S+\frac{1}{\mu}\left\langle\frac{1}{s}-\frac{1}{\mu+s}\right\rangle-\left\langle\frac{S}{\mu+s}\right\rangle \\
& =\left(S+\frac{1}{\mu}\right)\left(1-\left\langle\frac{1}{\mu+s}\right\rangle\right)=S+\frac{1}{\mu}-\frac{x(\mu)}{\mu}
\end{aligned}
$$

whence $x(\mu)>1$. When $\max \left(-1 / S, \max \left\{-s_{x}, x \in X\right\}\right)<\mu<0$, on the other hand, we get $y(\mu)<0$, from which $\left(S+\frac{1}{\mu}\right) x(\mu)<S+\frac{1}{\mu}$, whence $(1+\mu S) x(\mu)>1+\mu S$, giving still $x(\mu)>1$. 
Assume now that $\mu<\min \left(-1 / S, \min \left\{-s_{x}, x \in X\right\}\right)$. Let $\mu \rightarrow-\infty$, then $y(-\infty)=S^{2}-S>$ 0 (which is, again, coherent with Kelly's strategy value $y(+\infty)=y(-\infty)>0$ ). The inequality E61 holds, whence $y(\mu)>0$, from which $\left(S+\frac{1}{\mu}\right) x(\mu)>S+\frac{1}{\mu}$ (see E62), and (since $\left.S+\frac{1}{\mu}>0\right), x(\mu)>1$ still.

Proof of Theorem 2. The second derivative of the curve has same sign (see (E58) as

$$
F(\mu):=\left\{(1+\mu) L_{\mu}-1\right\}\left\{S-(1+S \mu) L_{\mu}\right\}
$$

Now,

$$
\begin{aligned}
F(\mu) & =-\frac{(1+\mu) L_{\mu}-1}{\mu}\left\{(1+S \mu)\left(1-\left\langle\frac{1}{s+\mu}\right\rangle\right)-S \mu\right\} \\
& =\left\{\frac{(1+\mu) L_{\mu}-1}{\mu}\right\}\left\{\left\langle\frac{1+S \mu}{s+\mu}\right\rangle-1\right\}
\end{aligned}
$$

We now use Lemma $1(2)$ and assume first that $\mu>0$. When $\mu>0,\left\langle\frac{1+S \mu}{s+\mu}\right\rangle>1$ so $F(\mu)>0$; thus that part of the front is strictly convex. Now (see eq. E17 and eq. E50), $\frac{1}{2} \frac{d \mu}{d m}=\frac{1}{2} \frac{d^{2}\left(\sigma_{W}^{2}\right)}{d m^{2}} \geq$ $\sigma_{W} \frac{d^{2} \sigma_{W}}{d m^{2}}>0$, whence $\frac{d\langle W\rangle}{d \mu}>0$. Starting from $\mu=0^{+}$and increasing $\mu$, one thus moves through the part of the lower front where $\langle W\rangle>0$.

Assume now that $\mu<\min \left(-1, \min \left\{-s_{x}, x \in X\right\}\right)$. Then $F(\mu)<0$; that part of the front is strictly concave. This holds true in particular in a neighborhood of Kelly's point on the upper front.

Assume finally that $0>\mu>\max \left(-1 / S, \max \left\{-s_{x}, x \in X\right\}\right)$. Then $F(\mu)<0$. That part of the front is strictly concave. This holds true in particular (as seen using the same argument as in the case $\mu>0$ ) in a neighborhood of the null strategy on the left, i.e. when $\langle W\rangle<0$.

\section{Appendix F: Horse race simulation}

For a given set of odds $o_{x}$, probabilities $p_{x}$ and gambler's betting strategy $b_{x}$, simulations represented in Fig. 1 in the main text have been performed by following these steps:

1. Simulation starts with unit capital $C_{0}=1$

2. A random number $r \in(0,1)$ is generated with a uniform distribution.

3. If $r<p_{1}$, horse number 1 wins this race. Otherwise, if $p_{1}<r<p_{1}+p_{2}$ then horse 2 wins this race. Otherwise, if $p_{1}+p_{2}<r<p_{1}+p_{2}+p_{3}$ horse 3 wins the race and so on. This assures that every horse wins a race according to the $p_{x}$ distribution.

4. Capital of the gambler is updated, according to the bets which have been placed and according to the odds given by bookmaker (i.e. using equation 1 in the main text).

5. Steps 2-4 are repeated for the number of desired races.

6. The log of the capital evolution is displayed in the figure.

The different lines in Figure 1 correspond to different realizations of the previous steps, with different betting strategies $b_{x}$ but the same odds and probabilities. We have simulated a race with three horses with $o_{1}=4\left(r_{1}=1 / 4\right), o_{2}=4\left(r_{2}=1 / 4\right)$, and $p_{1}=0.1, p_{2}=0.3$. 


\section{Appendix G: Numerical optimization}

To find the optimal bets $b^{*}$ for every objective function and parameter values, we use a simulated annealing algorithm, useful for global optimization problems in large search spaces, specially to avoid local maxima solutions. Starting from an initial guess $b_{0}$, a neighboring valid bets vector is randomly generated. It is accepted with probability 1 if it produces an increase in the value of the objective function $J$, that is, an "uphill" move. If not, then it is only accepted with probability proportional to an exponential factor $\exp (-\beta J)$, where $\beta$ plays the role of an inverse temperature. The algorithm is then repeated, increasing the value of $\beta$ in each iteration. In the beginning, with a small value for $\beta$ "uphill" moves are allowed, although with less probability than "downhill" moves, which avoids getting trapped in a local maximum. After a number of iterations, a stable value for the bets vector is obtained that gives an approximation to the optimal one.

As explained in the main text, there is a phase transition in this model where the optimal strategy changes from the null strategy to a mixed strategy when $\gamma$ approaches $\gamma_{c}$. Because of this transition, the Pareto front near the null strategy has a triangular shape, with a slope determined by this critical $\gamma_{c}$ as shown in Fig. 5 .

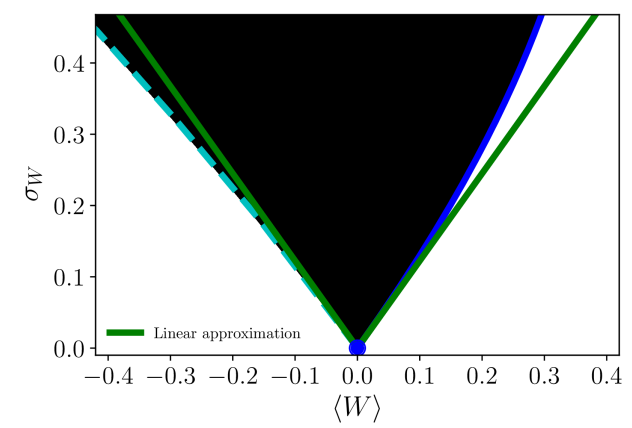

Figure 5: Zoom of the Pareto front shown in Fig 3 of the main text near the null strategy. The green solid lines correspond to the linear approximation used near the null strategy and the black points forming a dense region inside the front have been randomly chosen just as in Fig 3 . The figure corresponds to the case of three horses in the uncorrelated case with parameters $p_{1}=0.2, p_{2}=0.4, r_{1}=$ $0.4, r_{2}=0.2$.

The last figure of the main text, namely Fig 4, represents the Pareto border for 3 horses in the correlated case. We provide here some details on the parameters used to make this figure. Let $P(i, j)=P(i \mid j)$ represents the conditional probabilities of horse $i$ winning provided horse $j$ won in the previous round. Then $P$ is 3 by 3 matrix, which we choose to be

$$
P=\left(\begin{array}{lll}
0.2 & 0.2 & 0.3 \\
0.4 & 0.5 & 0.4 \\
0.4 & 0.3 & 0.3
\end{array}\right)
$$

The odds of the first two horses are $o_{1}=4\left(r_{1}=1 / 4\right), o_{2}=4\left(r_{2}=1 / 4\right)$, while the parameters of the last horse are deduced by normalization of the bets and of the probabilities. 